\title{
THE INFLUENCE ON A FINITE GROUP OF THE COFACTORS AND SUBCOFACTORS OF ITS SUBGROUPS
}

\author{
BY \\ LARRY R. NYHOFF( $\left.{ }^{1}\right)$
}

\begin{abstract}
The effect on a finite group $G$ of imposing a condition $\theta$ on its proper subgroups has been studied by Schmidt, Iwasawa, Itô, Huppert, and others. In this paper, the effect on $G$ of imposing $\theta$ on only the cofactor $H / \operatorname{cor}_{G} H$ (or more generally, the subcofactor $H / \operatorname{scor}_{G} H$ ) of certain subgroups $H$ of $G$ is investigated, where $\operatorname{cor}_{G} H$ (scor $\left.H\right)$ is the largest $G$-normal ( $G$-subnormal) subgroup of $H$. It is shown, for example, that if (a) $H / \operatorname{scor}_{G} H$ is $p$-nilpotent for all self-normalizing $H<G$, or if (b) $H /$ scor $_{G} H$ is $p$-nilpotent for all abnormal $H<G$ and $p$ is odd or the $p$-Sylows of $G$ are abelian, then in either case, $G$ has a normal $p$-subgroup $P$ for which $G / P$ is $p$-nilpotent. Results of this type are also derived for $\theta=$ nilpotent, nilpotent of class $\leqq n$, solvable of derived length $\leqq n, \sigma$-Sylow-towered, supersolvable. In some cases, additional structure in $G$ is obtained by imposing $\theta$ not only on these "worst" parts of the "bad" subgroups of $G$ (from the viewpoint of normality), but also on the "good" subgroups, those which are normal in $G$ or are close to being normal in that their cofactors are small.

Finally, this approach is in a sense dualized by an investigation of the influence on $G$ of the outer cofactors of its subgroups. The consideration of nonnormal outer cofactors is reduced to that of the usual cofactors. The study of normal outer cofactors includes the notion of normal index of maximal subgroups, and it is proved, for example, that $G$ is $p$-solvable iff the normal index of each abnormal maximal subgroup of $G$ is a power of $p$ or is prime to $p$.
\end{abstract}

There are a number of theorems which describe the effect on a finite group $G$ of a condition $\theta$ imposed on its proper subgroups. For example, Schmidt [15] and Iwasawa [12] have shown that if every proper subgroup of a finite group $G$ is nilpotent, then $G$ is solvable, and among other things (see Theorem 2-A), if $G$ itself is nonnilpotent, then $|G|=p^{a} q^{b}$ for distinct primes $p$ and $q, G$ has a normal $p$-Sylow subgroup and cyclic $q$-Sylow subgroups. Huppert [10] and Doerk [5] have obtained corresponding results for the case where the proper subgroups of $G$ are

Received by the editors April 16, 1970.

AMS subject classifications. Primary 2025, 2040.

Key words and phrases. Core, subnormal core, cofactor, subnormal cofactor, solvable group, nilpotent group, Schmidt-Iwasawa Theorem, abnormal subgroup, self-normalizing subgroup, Fitting subgroups, nearly normal subgroups, $p$-nilpotent and $p$-solvable groups, Itô's Theorem, Thompson subgroup, Glaubermann-Thompson Theorem, $p$-complement, somewhat normal subgroup, $(p: q)$-nilpotent group, $\sigma$-Sylow-towered, supersolvable groups, outer cofactors, self-normalizing and abnormal outer cofactors, normal index.

( $\left.{ }^{1}\right)$ The major part of this paper is a portion of the author's Ph.D. dissertation written at Michigan State University under the direction of Professors J. E. Adney and W. E. Deskins, while the author was a National Science Foundation Science Faculty Fellow. 
supersolvable. Results are also known for the cases where the proper subgroups are $p$-nilpotent, or abelian, or $\sigma$-Sylow-towered.

Extensions of such results have been obtained by imposing $\theta$ on only certain subgroups of $G$. For example, Deskins [3] has shown that the finite group $G$ is solvable if it has a nilpotent maximal subgroup having Sylow subgroups of class $\leqq 2$. Rose [14] has established: (1) If all the proper abnormal subgroups of $G$ are nilpotent, then $G$ is solvable and has a normal Sylow subgroup $P$ such that $G / P$ is nilpotent. (2) If all the proper self-normalizing subgroups of $G$ are supersolvable, then $G$ is solvable.

As in these last two results, the chief concern here is with the effect on a finite group $G$ of a condition $\theta$ imposed on the "bad" subgroups of $G$ (from the viewpoint of normality), namely, the self-normalizing, or the abnormal, subgroups. However, we will not require that $\theta$ be satisfied by these subgroups themselves, but only by their "worst parts" (again from the viewpoint of normality or subnormality), that is, by their cofactors or subcofactors. In some cases, to obtain more structure in $G$, we will impose $\theta$ on the "good" subgroups of $G$ also, those which are normal in $G$, or are rather close to being normal in the sense that their cofactors are quite small.

1. Preliminaries. For $H$ a subgroup of a finite group $G$, the core of $H$ in $G$, $\operatorname{cor}_{G} H$, is the largest $G$-normal subgroup of $H$; alternatively, $\operatorname{cor}_{G} H=\bigcap_{x \in G} H^{x}$; the subnormal core of $H$ in $G$, scor $_{G} H$, is the largest $G$-subnormal subgroup of $H$. It is well known that for $G$ finite, if $L_{1}$ and $L_{2}$ are subnormal in $G$, then so also is $\left\langle L_{1}, L_{2}\right\rangle$; thus, $\operatorname{scor}_{G} H$ is well defined for $G$ finite.

Lemma 1.1. For $H$ a subgroup of the finite group $G, \operatorname{scor}_{G} H$ is normal in $H$.

Proof. We have $L=\operatorname{scor}_{G} H \triangleleft \triangleleft G$, say $L \triangleleft N_{1} \triangleleft \cdots \triangleleft N_{r}=G$. Let $x$ be any element of $H$; clearly, $L^{x} \triangleleft N_{1}^{x} \triangleleft \cdots \triangleleft N_{r}^{x}=G$, hence $L^{x} \triangleleft \triangleleft G$. Thus, $\left\langle L, L^{x}\right\rangle$ is subnormal in $G$ and is contained in $H$, hence is contained in $\operatorname{scor}_{G} H=L$ so that $L^{x}=L$.

For $H$ a subgroup of the finite group $G$, the cofactor of $H$ in $G$, denoted $\operatorname{cof}_{G} H$, is defined as $H / \operatorname{cor}_{G} H$; in view of Lemma 1.1, the subnormal cofactor of $H$ in $G$, $\operatorname{scof}_{G} H$, can be defined as $H /$ scor $_{G} H$.

All groups considered here are assumed to be finite. It might be mentioned, however, that the definitions of $\operatorname{cor}_{G} H$ and $\operatorname{cof}_{G} H$ are still legitimate in case $G$ is infinite. Also, it follows from Poincaré's Lemma (see Theorems 1.1.10 and 3.3.5 in [16]) that if $G$ has a proper subgroup $K$ of finite index, then $G / \operatorname{cor}_{G} K$ is a finite group. Using this allows one to apply the results given here to obtain information about such infinite groups. For example, it follows from (i) of Lemma 1.3 (which holds for all groups) and Theorem 2.6 that if $G$ has a proper subgroup $K$ of finite index and if the proper normal subgroups of $G$ as well as the cofactors of maximal subgroups of $G$ are nilpotent, then $G$ is solvable and the conclusions of Theorem 2.6 hold for $G / \operatorname{cor}_{G} K$.

It is obvious from the definitions for $H<G, \operatorname{cor}_{G} H \subseteq \operatorname{scor}_{G} H$, so $\operatorname{scof}_{G} H$ 
$=H / \operatorname{scor}_{G} H$ is a homomorphic image of $\operatorname{cof}_{G} H=H / \operatorname{cor}_{G} H$. It follows that any result obtained from imposing a homomorphism-invariant condition $\theta$ on the subcofactors of subgroups of $G$ will automatically hold if the cofactors of these subgroups are $\theta$-groups. Thus, wherever possible, we will impose conditions on the subcofactors of subgroups as opposed to their cofactors.

In general, $\operatorname{scor}_{G} H \neq \operatorname{cor}_{G} H$; for example, if $H$ is a subgroup of order 2 in $G=A_{4}=$ the alternating group of degree 4 , then $\operatorname{cor}_{G} H=1$ and $\operatorname{scor}_{G} H=H$. For maximal subgroups, however, the core and subnormal core must coincide.

LEMMA 1.2. If $S$ is a maximal subgroup of the finite group $G$, then $\operatorname{cor}_{G} S=\operatorname{scor}_{G} S$.

Proof. Let $\operatorname{scor}_{G} S \underset{\ddagger}{\triangleleft} N_{1 \neq} N_{2} \sharp \cdots \supsetneqq N_{r}=G$. Since $\operatorname{scor}_{G} S$ is the maximal $G$ subnormal subgroup of $S, N_{1} \nsubseteq S$ since $N_{1} \triangleleft \triangleleft G$. From Lemma 1.1, $\operatorname{scor}_{G} S \triangleleft S$; thus, since $\operatorname{scor}_{G} S \triangleleft N_{1}$, we have $\operatorname{scor}_{G} S \triangleleft\left\langle N_{1}, S\right\rangle=G$ so that $\operatorname{scor}_{G} S \subseteq \operatorname{cor}_{G} S$. Since the reverse inclusion is obvious, the desired equality follows.

The next lemma is essential for induction arguments. It follows immediately from the definitions and the isomorphism theorems.

LEMMA 1.3. Given $G$ finite, $K \triangleleft G$, and $K \subseteq H<G$. Then

(i) $\operatorname{cor}_{G / K}(H / K)=\operatorname{cor}_{G} H / K$, and $\operatorname{cof}_{G / K}(H / K) \simeq \operatorname{cof}_{G} H$;

(ii) $\operatorname{scor}_{G / K}(H / K)=\operatorname{scor}_{G} H / K$, and $\operatorname{scof}_{G / K}(H / K) \simeq \operatorname{scof}_{G} H$.

For $\theta$ a group-theoretic property, Baer [1] defines the $\theta$-commutator subgroup of $G,[G, \theta]$, as $\bigcap\{K \triangleleft G \mid G / K$ is a $\theta$-group $\}$. $\theta$ is said to be strictly homomorphisminvariant if (a) $\theta$ is homomorphism-invariant; (b) $\theta$ is subgroup-inherited; (c) $G /[G, \theta]$ is a $\theta$-group. In the presence of (a) and (b), condition (c) is equivalent to $\left(c^{\prime}\right)$ direct products of $\theta$-groups are $\theta$-groups. $\theta=$ abelian, nilpotent, $p$-nilpotent, supersolvable, solvable are obviously strictly homomorphism-invariant properties.

THEOREM 1-A. Let $\theta$ be a strictly homomorphism-invariant property, and let $G$ be a finite group.

(i) (Baer) If $\theta$-groups are nilpotent, then $[G, \theta]$ is nilpotent if and only if $\operatorname{cof}_{G} S$ is a $\theta$-group for all maximal subgroups $S$ of $G$.

(ii) (Baer) $[G, \theta]$ is nilpotent and $G /[G, \theta]$ is solvable if and only if $\operatorname{cof}_{G} S$ is a $\theta$-group for all maximal subgroups $S$ of $G$ and equicore maximal subgroups of $G$ are conjugate in $G$.

(iii) (Ore) For $G$ solvable, equicore maximal subgroups of $G$ are conjugate in $G$.

Using the Fitting subgroup of $G, F(G)=$ the largest normal nilpotent subgroup of $G$, we have the following immediate corollary.

COROLlaRY 1.4. Let $\theta$ be a strictly homomorphism-invariant property and let $G$ be a finite group.

(i) If $\theta$-groups are nilpotent, then $G / F(G)$ is a $\theta$-group if and only if $\operatorname{cof}_{G} S$ is a $\theta$-group for all maximal subgroups $S$ of $G$.

(ii) If $G$ is solvable, then $G / F(G)$ is a $\theta$-group if and only if $\operatorname{cof}_{G} S$ is a $\theta$-group for all maximal subgroups $S$ of $G$. 
2. $\theta=$ nilpotent. In this section we examine the influence on a group $G$ of nilpotent subcofactors of subgroups of $G$, and investigate what additional structure is forced upon $G$ if one then also requires that the proper normal subgroups be nilpotent, and finally, that the nearly normal maximal subgroups be nilpotent. The classic result in this direction is the following Schmidt-Iwasawa Theorem ([15], [12], Satz 5.2 of [9, p. 281]).

THEOREM 2-A. If all proper subgroups of the finite group $G$ are nilpotent, but $G$ itself is nonnilpotent, then (i) $|G|=p^{a} q^{b}$ for distinct primes $p$ and $q$; (ii) $G$ has a normal p-Sylow subgroup $P$; (iii) $\Phi(P) \subseteq Z(G)$, so $P$ has class $\leqq 2$; (iv) $\exp P=p$ or $\exp P \leqq 4$ according as $p$ is odd or $p=2 ;(\mathrm{v})$ each $q$-Sylow subgroup $Q$ of $G$ is cyclic and $\Phi(Q) \subseteq Z(G)$.

In this same direction, Rose [14] has shown the following:

THEOREM 2-B. If all the proper abnormal subgroups of the finite group $G$ are nilpotent, then $G$ has a normal Sylow subgroup $P$ such that $G / P$ is nilpotent.

Here a subgroup $H$ is abnormal in $G$, denoted $H \rtimes G$, if $x \in\left\langle H, H^{x}\right\rangle$ for each $x \in G$, or equivalently, if every subgroup $K$ of $G$ containing $H$ is self-normalizing in $G$ (that is, $N_{G}(K)=K$ ) and $H$ is not contained in two distinct conjugate subgroups of $G$. For example,

(i) $N_{G}(P) \rtimes G$ for every Sylow subgroup $P$ of $G$.

(ii) If $H \rtimes G$ and $H \subseteq K<G$, then $K \rtimes G$.

(iii) A maximal subgroup $S$ of $G$ is abnormal in $G$ if and only if $S$ is selfnormalizing in $G$ if and only if $S$ is nonnormal in $G$.

Since nilpotency is a strictly homomorphism-invariant property, we have the following as an immediate consequence of Corollary 1.4.

THeOREM 2.1. For $G$ a finite group, $G / F(G)$ is nilpotent if and only if $\operatorname{cof}_{G} S$ $=S / \operatorname{cor}_{G} S$ is nilpotent for all maximal subgroups $S$ of $G$. Alternatively, the Fitting length of $G, f(G)$, is at most 2 if and only if $\operatorname{cof}_{G} S$ is nilpotent for all maximal subgroups $S$ of $G$.

Here $f(G)$ is the least integer $n$ for which $F_{n}(G)=G$, where $1=F_{0}(G) \subseteq F_{1}(G) \subseteq \cdots$ is the ascending Fitting series of $G$ defined by $F_{i+1}(G) / F_{i}(G)=F\left(G / F_{i}(G)\right)=$ the Fitting subgroup of $G / F_{i}(G)$.

One would hope to obtain more structure in $G$ by requiring that the cofactors of maximal subgroups are all nilpotent of the same class. The following results describe what can be said in this case.

LEMma 2.2. Let $\Gamma_{n}$ denote the property "nilpotent of class $\leqq n$ ". Then

(i) For any group $G$, the $\Gamma_{n}$-commutator subgroup $\left[G, \Gamma_{n}\right]=\gamma_{n}(G)$, where $G=$ $\gamma_{0}(G) \supseteq \gamma_{1}(G) \supseteq \cdots$ is the descending central series of $G$.

(ii) $\Gamma_{n}$ is a strictly homomorphism-invariant property. 
Proof. (i)

$$
\begin{aligned}
{\left[G, \Gamma_{n}\right] } & =\bigcap\left\{K \triangleleft G \mid G / K \text { is a } \Gamma_{n} \text {-group }\right\} \\
& =\bigcap\left\{K \triangleleft G \mid \gamma_{n}(G / K)=1\right\} \\
& =\bigcap\left\{K \triangleleft G \mid \gamma_{n}(G) K / K=1\right\} \\
& =\bigcap\left\{K \triangleleft G \mid \gamma_{n}(G) \subseteq K\right\}=\gamma_{n}(G) .
\end{aligned}
$$

(ii) $\Gamma_{n}$ is clearly homomorphism-invariant and subgroup-inherited. The fact that $G /\left[G, \Gamma_{n}\right]$ is a $\Gamma_{n}$-group follows immediately from (i).

THEOREM 2.3. For $G$ a finite group, the following are equivalent:

(i) $G / F(G)$ is nilpotent of class $\leqq n$.

(ii) $\operatorname{cof}_{G} S=S / \operatorname{cor}_{G} S$ is nilpotent of class $\leqq n$ for all maximal subgroups $S$ of $G$.

(iii) $\gamma_{n}(G)$ is nilpotent.

(iv) $\gamma_{n}(H) \triangleleft \triangleleft G$ for all subgroups $H$ of $G$.

(v) $\gamma_{n}(H) \triangleleft \triangleleft G$ for all abnormal subgroups $H$ of $G$.

Proof. The equivalence of (i) and (ii) is an immediate consequence of Lemma 2.2 and Corollary 1.4; (ii) $\rightarrow$ (iii) follows from Lemma 2.2 and Theorem 1-A.

(iii) $\rightarrow$ (iv). If $H<G$, then since $\gamma_{n}(H) \subseteq \gamma_{n}(G)$ and $\gamma_{n}(G)$ is nilpotent, $\gamma_{n}(H) \triangleleft \triangleleft$ $\gamma_{n}(G) \triangleleft G$ so that $\gamma_{n}(H) \triangleleft \triangleleft G$.

(iv) $\rightarrow$ (v) is trivial.

(v) $\rightarrow$ (ii). Let $S$ be any maximal subgroup of $G$. Since $\operatorname{cof}_{G} S=1$ if $S \triangleleft G$, we may assume $S \Varangle G$ and thus $S \rtimes G$. Then since $\gamma_{n}(S) \triangleleft \triangleleft G$, we have, using Lemma $1.2, \gamma_{n}(S) \subseteq \operatorname{scor}_{G} S=\operatorname{cor}_{G} S$. Thus $\operatorname{cof}_{G} S=S / \operatorname{cor}_{G} S$ is a homomorphic image of the $\Gamma_{n}$-group $S / \gamma_{n}(S)$ and hence is itself nilpotent of class $\leqq n$.

In a similar way one can show that the property $\Delta_{n}=$ "solvable of derived length $\leqq n$ " is a strictly homomorphism-invariant property and that $\left[G, \Delta_{n}\right]=G^{(n)}$, where $G=G^{(0)} \supseteq G^{(1)} \supseteq \cdots$ is the derived series of $G$. This leads to the following:

THEOREM 2.4. For $G$ a finite solvable group, the following are equivalent:

(i) $G / F(G)$ has derived length $\leqq n$.

(ii) $\operatorname{cof}_{G} S=S / \operatorname{cor}_{G} S$ has derived length $\leqq n$ for all maximal subgroups $S$ of $G$.

(iii) $G^{(n)}$ is nilpotent.

(iv) $H^{(n)} \triangleleft \triangleleft G$ for all subgroups $H$ of $G$.

(v) $H^{(n)} \triangleleft \triangleleft G$ for all abnormal subgroups $H$ of $G$.

Imposing the condition of nilpotence or nilpotence of class at most $n$ on the cofactors of the maximal subgroups of $G$ is not sufficient to guarantee that $G$ has a normal Sylow subgroup; in particular, the conclusions of Theorems 2-A and 2-B need not hold. In fact, it is not sufficient to require that the cofactors of all proper subgroups be abelian. The following example shows this and can be verified in a straightforward manner.

EXAmple 2.5. The group $G=S_{3} \times A_{4}$, where $S_{3}$ is the symmetric group on 3 letters and $A_{4}$ is the alternating group of degree 4, has no normal Sylow subgroups, but $H / \operatorname{cor}_{G} H$ is abelian for all proper subgroups $H$ of $G$. 
If we now require that in addition to the cofactors of the maximal subgroups of $G$ being nilpotent, the proper normal subgroups of $G$ also are nilpotent, we would surely hope to find more structure in $G$. Although Example 2.8 shows that we cannot recover all of the results of Schmidt, Iwasawa, and Rose, we do find the structure of $G$ is quite severely restricted. The following result spells out some of this structure; here we will say that a subgroup $H$ of $G$ is nearly normal in $G$ if $\operatorname{cof}_{G} H=H / \operatorname{cor}_{G} H$ is trivial or of prime order, and that $H$ is nearly subnormal in $G$ if $\operatorname{scof}_{G} H=H / \operatorname{scor}_{G} H$ is trivial or of prime order.

THEOREM 2.6. Let $|G|=\prod_{i=1}^{r} p_{i}^{a_{i}}$, where the $p_{i}$ are distinct primes dividing $|G|$. $S$ uppose that $\operatorname{cof}_{G} S$ is nilpotent for all maximal subgroups $S$ of $G$ and that all proper normal subgroups of $G$ are nilpotent, but that $G$ itself is nonnilpotent. Then $G$ is solvable and $F(G)$ is the unique maximal normal subgroup of $G$. Moreover, for one of the prime factors of $|G|$, say $p_{r}$, the following hold:

(1) $G$ is $p_{r}$-nilpotent, that is, $G$ has a normal $p_{r}$-complement.

(2) $G$ is non-p $p_{i}$-nilpotent for all $i \neq r$.

(3) $[G: F(G)]=p_{r}$.

(4) For all $H<G,\left|\operatorname{scof}_{G} H\right|=1$ or $p_{r}$; in particular, all proper subgroups of $G$ are nearly subnormal in $G$, and all maximal subgroups are nearly normal in $G$.

(5) For each $i \neq r, G$ has a normal $p_{i}$-Sylow subgroup $P_{i}$.

(6) For each $i \neq r, P_{i} \subseteq G^{\prime}$; thus $G / G^{\prime}$ is a $p_{r}$-group.

(7) The prime $p_{r}$ divides $d_{p_{1}}=\prod_{j=1}^{a_{i}}\left(p_{i}^{j}-1\right)$ for all $i \neq r$.

(8) Each $p_{r}$-Sylow subgroup $Q$ has $G$ as its normal closure, that is, $Q^{G}=G$.

(9) For each $i \neq r$ for which $P_{i}$ is abelian: (i) $G$ has exactly $p_{i}^{a_{i}}$ distinct $p_{i}$-complements; (ii) $C_{G}\left(P_{i}\right)=F(G)$, so $G$ induces in $P_{i}$ a group of automorphisms of order $p_{r}$; (iii) the number of $p_{r}$-Sylow subgroups of $G$ is a multiple of $p_{i}^{a_{i}}$.

(10) If $P_{i}$ is abelian for all $i \neq r$, then $G$ has exactly $|G| / p_{r}^{a_{r}}$ distinct $p_{r}$-Sylow subgroups, each of which is abnormal in $G$, and which are the system normalizers (=Carter subgroups) of $G$.

Proof. The solvability of $G$ follows from Theorem 2.3; the fact that $F(G)$ is the unique maximal normal subgroup is trivial, since all proper normal subgroups of $G$ are nilpotent, hence are contained in $F(G)$.

(3) is immediate, since maximal normal subgroups in a solvable group have prime index; relabelling if necessary, we may take $[G: F(G)]=p_{r}$.

(1) $F(G)$ is nilpotent, hence $p_{r}$-nilpotent. Its normal $p_{r}$-complement $T$ is characteristic in $F(G)$, thus is normal in $G$, and since $[G: F(G)]=p_{r}, T$ is a normal $p_{r^{-}}$ complement of $G$.

(4) Let $H$ be any proper subgroup of $G$. If $H \subseteq F(G)$, then by the nilpotence of $F(G), H \triangleleft \triangleleft F(G) \triangleleft G$, and hence, $\operatorname{scor}_{G} H=H$. So suppose $H \nsubseteq F(G)=F$. Then $H F=G$, so $|H \cap F|=|H||F| /|H F|=(|H| /|G|)\left(|G| / p_{r}\right)=|H| / p_{r}$. How $H \cap F \triangleleft \triangleleft$ $F \triangleleft G$ which means that $H \cap F \subseteq \operatorname{scor}_{G} H$. Thus, since $[H: H \cap F]=p_{r}, H /$ scor $_{G} H$ 
has order 1 or $p_{r}$. In particular, if $S$ is a maximal subgroup of $G$, then since $\operatorname{scor}_{G} S=\operatorname{cor}_{G} S$, we have $\left|S / \operatorname{cor}_{G} S\right|=1$ or $p_{r}$ so that $S$ is nearly normal in $G$.

(5) is immediate; for since $[G: F(G)]=p_{r}$, the $p_{i}$-Sylow subgroups of $F(G)$ for $i \neq r$ are $p_{i}$-Sylow subgroups of $G$ and are characteristic in $F(G)$, hence are normal in $G$.

(2) If for some $i \neq r, G$ is $p_{i}$-nilpotent, say with normal $p_{i}$-complement $T_{i}$, then $T_{i}$, being nilpotent, would have a characteristic $p_{r}$-Sylow subgroup $Q$ which would then be a normal $p_{r}$-Sylow subgroup of $G$. But this together with (5) would imply that $G$ is nilpotent.

(6) Letting $G_{\left(p_{i}\right)}^{\prime}$ be the smallest normal subgroup of $G$ for which the factor group is an abelian $p_{i}$-group, we note first that $G_{\left(p_{i}\right)}^{\prime}=G$ for each $i \neq r$. If not, it would be nilpotent by hypothesis, hence $p_{i}$-nilpotent, and its characteristic $p_{i}$-complement would then be a normal $p_{i}$-complement of $G$, in contradiction to (2). It now follows from one of the basic transfer theorems (see $[16,13.5 .2]$ ) that $1=G / G_{\left(p_{i}\right)}^{\prime} \simeq P_{i} / P_{i} \cap G^{\prime}$ so that $P_{i} \subseteq G^{\prime}$ for each $i \neq r$.

(7) Case 1. For some $i \neq r, P_{i}$ is not minimal normal in $G$.

Let $M \neq 1$ be a normal subgroup of $G$ properly contained in $P_{i}$. Then $G / M$ is not $p_{i}$-nilpotent; for if it were, say with normal $p_{i}$-complement $T / M$, then $T$, being a proper normal subgroup of $G$, would be nilpotent, hence $p_{i}$-nilpotent, and its characteristic $p_{i}$-complement would then be a normal $p_{i}$-complement of $G$ in contradiction to (2). Also, the other hypotheses hold for $G / M$ so that by induction, for each $i \neq r, p_{r}$ divides $d_{p_{i}}^{*}=\prod_{j=1}^{b_{i}}\left(p_{i}^{j}-1\right)$, where $|G / M|=\prod_{i=1}^{r} p_{i}^{b_{i}}$. Hence, $p_{r}$ also divides $d_{p_{t}}=\prod_{j=1}^{a_{i}}\left(p_{i}^{j}-1\right)$ for each $i \neq r$.

Case 2. $a_{r}>1$.

Let $Q$ be a $p_{r}$-Sylow subgroup of $G$. From (4), $\left|Q / \operatorname{cor}_{G} Q\right|=p_{r}$, so since $a_{r}>1$, $\operatorname{cor}_{G} Q \neq 1$. Now, $G / \operatorname{cor}_{G} Q$ is non- $p_{i}$-nilpotent for $i \neq r$; otherwise, if $T / \operatorname{cor}_{G} Q$ were a normal $p_{i}$-complement, $T$ would be a normal $p_{i}$-complement of $G$ in contradiction to (2). Again, the other hypotheses hold for $G / \operatorname{cor}_{G} Q$ so that, by induction, since all the $p_{i}$ divide $\left|G / \operatorname{cor}_{G} Q\right|$, we have the result as in Case 1 .

Case 3. $a_{r}=1$ and, for all $i \neq r, P_{i}$ is minimal normal in $G$.

Then each $P_{i}, i \neq r$, is elementary abelian so that $\mid$ Aut $\left(P_{i}\right) \mid=p_{i}^{p_{i}} \cdot d_{p_{i}}$, where $e_{i}=a_{i}\left(a_{i}-1\right) / 2$ and $d_{p_{i}}$ is as in the theorem. Now from (9), which will be proved independently, $C_{G}\left(P_{i}\right)=F(G)$ for $i \neq r$, so $\left[G: C_{G}\left(P_{i}\right)\right]=p_{r}$. Thus, for $i \neq r, p_{r}$ divides $\mid$ Aut $\left(P_{i}\right) \mid$, and hence divides $d_{p_{i}}$.

(8) is immediate, since if for some $p_{r}$-Sylow subgroup $Q, Q^{G} \neq G$, then $Q^{G}$ would be nilpotent by hypothesis, making $Q \triangleleft G$. But this together with (5) would contradict the nonnilpotence of $G$.

(9) (i) is an immediate consequence of Theorem 3.4 to be proved later.

(ii) If $P_{i}$ is abelian for some $i \neq r$, then $F(G)$ centralizes $P_{i}$ so that $C_{G}\left(P_{i}\right)=F(G)$ or $G$, by the maximality of $F(G)$. However, $P_{i} \notin Z(G)$; otherwise, $P_{i}$ would centralize and hence normalize a $p_{i}$-complement $T_{i}$ of $G$ making $T_{i} \triangleleft G$, which contradicts (2). Therefore, $C_{G}\left(P_{i}\right)=F(G)$. 
(iii) If $Q$ is any $p_{r}$-Sylow subgroup of $G$, then $Q W$ is a $p_{i}$-complement of $G$ containing $Q$, where $W$ is the unique $p_{i}$-complement of $F(G)$; thus,

(a) each $p_{r}$-Sylow subgroup of $G$ is contained in some $p_{i}$-complement of $G$ for each $i \neq r$.

Also, if $X$ and $Y$ are two distinct $p_{i}$-complements of $G$, then $X \cap F(G)=W$ $=Y \cap F(G),[X: W]=[Y: W]=p_{r}$, so $X \cap Y=W$; and since $p_{r}^{a_{r}}$ does not divide $|W|=|X \cap Y|$, it follows that

(b) no two distinct $p_{i}$-complements of $G$ have a $p_{r}$-Sylow subgroup of $G$ in common.

Since $G$ is solvable,

(c) any two $p_{i}$-complements are conjugate in $G$ and hence must contain the same number, say $\mu$, of $p_{r}$-Sylow subgroups of $G$.

Finally, from (i), we have that if $P_{i}$ is abelian,

(d) $G$ has exactly $p_{i}^{a_{i}}$ distinct $p_{i}$-complements.

Statements (a)-(d) imply that $G$ has exactly $\mu p_{i}^{a_{i}}$ distinct $p_{r}$-Sylow subgroups, if $P_{i}$ is abelian.

(10) If $n=$ the number of $p_{r}$-Sylow subgroups of $G$, then since $P_{i}$ is abelian for all $i \neq r$, we have from $\left(9(\right.$ iii) $)$ that $p_{i}^{a_{i}} \mid n$ for all $i \neq r$. Since $n \equiv 1\left(\bmod p_{r}\right)$, it follows that $n=|G| / p_{r}^{a_{r}}$. Each $p_{r}$-Sylow subgroup $Q$ is abnormal in $G$ since $N_{G}(Q) \rtimes G$, and by what we have just shown, $N_{G}(Q)=Q$.

Since $F(G)$ is nilpotent as is $G / F(G)$, the Carter subgroups of $G$ coincide with the system normalizers. From (1) and (9(i)), it follows that $G$ has $|G| / p_{r}^{a_{r}}$ distinct Sylow systems, and hence has at most $|G| / p_{r}^{a_{r}}$ distinct system normalizers. But from (9(iii)), each $p_{r}$-Sylow subgroup is a Carter subgroup, hence a system normalizer; thus, since $G$ has exactly $|G| / p_{r}^{a_{r}} p_{r}$-Sylow subgroups, these must be all the system normalizers of $G$.

Statement (4) of the preceding theorem shows that if we extend the requirement of nilpotence to the nearly normal maximal subgroups of $G$, then all maximal subgroups are nilpotent so that Theorem 2-A holds, giving the following corollary.

COROLLARY 2.7. If the cofactors of all maximal subgroups of $G$ are nilpotent as are the nearly normal maximal subgroups of $G$, but $G$ itself is nonnilpotent, then all proper subgroups of $G$ are nilpotent, and thus, the conclusions of Theorem 2-A hold (as do those of Theorem 2.6).

The condition imposed in Theorem 2.6 that a nonnilpotent group have the cofactors of its maximal subgroups and all its proper normal subgroups nilpotent does not, in contrast to the Schmidt-Iwasawa Theorem, impose any bounds on $|\pi(G)|$. Neither does it guarantee that $G$ has a normal Sylow subgroup for which the factor group is nilpotent, so Theorem 2-B cannot be recovered. In fact, it is not even sufficient to require that the cofactors of all subgroups and the proper normal subgroups be cyclic, as the following example shows. 
EXAMPLE 2.8. For all $n \geqq 3$, there exists a nonnilpotent group $G$ such that

(i) The cofactors of all subgroups of $G$ and the proper normal subgroups of $G$ are cyclic.

(ii) $|G|$ is divisible by $n$ distinct primes.

(iii) $G$ has no normal Sylow subgroup $P$ for which $G / P$ is nilpotent.

Proof. Take any collection $\Sigma=\left\{p_{1}, \ldots, p_{n-1}\right\}$ of $n-1$ distinct odd primes, and for each $i$, let $P_{i}=\left\langle x_{i}\right\rangle$ be cyclic of order $p_{i}$ and $\alpha_{i}$ an automorphism of $P_{i}$ of order 2 . Let $K=P_{1} \times \cdots \times P_{n-1}$ and $G$ the extension of $K$ by the automorphism $\alpha=\alpha_{1} \times \cdots$ $\times \alpha_{n-1}$. Then $G$ is not nilpotent; for otherwise, $\langle\alpha\rangle$ would be normal in $G$, hence would centralize $K$ in contradiction to the fact that $\alpha$ has order 2. This group $G$ clearly satisfies (ii), so only (i) and (iii) require proof.

(i) Let $H$ be any proper subgroup of $G$. If $2 \nmid|H|$, then clearly $H$ is cyclic. Thus we may assume that 2||$H \mid$. Then $H$ is not normal in $G$. For suppose it is. Since $H \neq G$, some $p_{t}$ does not divide $|H|$, so $P_{t} \cap H=1$. Also, the 2-Sylow subgroups of $G$ are contained in $H$, in particular, $\alpha \in H$. Then $\left[x_{t}, \alpha\right]=x_{t}^{-1} \alpha x_{t} \alpha$ is in $H$. But since $P_{t}=\left\langle x_{t}\right\rangle$ is characteristic in $G,\left[x_{t}, \alpha\right] \in P_{t}$. Thus $\left[x_{t}, \alpha\right] \in P_{t} \cap H=1$, which implies that $x_{t}^{\alpha}=x_{t}$, contradicting the fact that $\alpha_{t}=\left.\alpha\right|_{P_{t}}$ has order 2. Therefore $H \nless G$; and it is clear that $\operatorname{cor}_{G} H$ is the product of the $P_{i}$ for those $p_{i}$ dividing $|H|$. Consequently, $H /$ cor $_{G} H$ is cyclic of order 2 .

(iii) The $P_{i}$ are the normal Sylow subgroups of $G$. If for some $i, G / P_{i}$ were nilpotent, then for a 2-Sylow subgroup $T$ of $G$, we have $T P_{i} / P_{i} \triangleleft G / P_{i}$, hence $T P_{i} \triangleleft G$, which contradicts the proof in (i) that if 2||$H \mid$, then $H \Varangle G$.

3. $\theta=p$-nilpotent. We now examine those finite groups $G$ for which the cofactors or subcofactors of certain subgroups are $p$-nilpotent, that is, they possess a normal $p$-complement. As in the preceding section, we will later require that the proper normal subgroups of $G$ also be $p$-nilpotent to further delimit the structure of $G$, and finally, that the somewhat normal subgroups be $p$-nilpotent. One of the major results in this direction is the following, due to Itô [11] (also proved in [9, p. 434, Satz 5.4]).

THEOREM 3-A. If all the proper subgroups of the finite group $G$ are p-nilpotent, but $G$ itself is not, then all proper subgroups of $G$ are nilpotent, and thus the conclusions of Theorem 2-A hold.

Rose [14] has also established results in this direction:

THEOREM 3-B. (i) If every proper self-normalizing subgroup of $G$ is p-nilpotent, then $G$ has a normal p-subgroup $P_{0}$ (which may be trivial) such that $G / P_{0}$ is p-nilpotent.

(ii) If every proper abnormal subgroup of $G$ is p-nilpotent and either $p$ is odd or the p-Sylow subgroups of $G$ are abelian, then the conclusion of (i) holds.

That the added conditions on $p$ in (ii) cannot be omitted is shown by the following example given by Rose in [14]: 
EXAMPLE 3-C. Let $H$ be the simple group of order $128(H=\operatorname{PGL}(3,2))$, and $G$ the split extension of $H$ by the automorphism $\alpha$ of $H$ defined by $\alpha: x \rightarrow\left(x^{-1}\right)^{t}$, where $y^{t}$ denotes the transpose of the matrix $y$. Then every proper abnormal subgroup of $G$ is supersolvable, hence 2-nilpotent, but $G$ is not solvable, hence not 2-solvable.

The results of Rose in Theorem 3-B can be extended by requiring that only the subcofactors of the self-normalizing or abnormal subgroups be $p$-nilpotent. To establish this, the following well-known results of Burnside (see [9, pp. 434, 419, Hilfssatz 5.3, Hauptsatz 2.6]) and the lemma which follows are used.

THEOREM 3-D. (i) If the finite group $G$ is not p-nilpotent, then $G$ has a nontrivial $p$-subgroup $P_{0}$ and an element $x$ of order a power of $q$ for some prime $q \neq p$ such that $x \in N_{G}\left(P_{0}\right)$ but $x \notin C_{G}\left(P_{0}\right)$.

(ii) If $G$ is a finite group with p-Sylow subgroup $P \subseteq Z\left(N_{G}(P)\right)$, then $G$ is p-nilpotent.

LEMMA 3.1. Let $\theta$ be a group-theoretic property such that products of normal $\theta$-subgroups of a group are again $\theta$-groups. If the finite group $G$ has a nontrivial subnormal $\theta$-subgroup, then $G$ has a nontrivial normal $\theta$-subgroup.

Proof. Let $K$ be a nontrivial $\theta$-subgroup of $G$ which is subnormal in $G$, say $K=K_{0 \neq}{ }_{1}{ }_{1} \cdots \rightrightarrows K_{n-1}{ }^{\unlhd} K_{n}=G$, where $n$ is the minimal length of subnormal chains from $K$ to $G$. We use induction on $n$. The result is trivially true if $n=1$; thus, suppose $n>1$. By induction, $K_{n-1}$ has a nontrivial normal $\theta$-subgroup; let $K^{*}$ be the product of all such. Then $K^{*}$ is a nontrivial $\theta$-group, is clearly characteristic in $K_{n-1}$, and hence is normal in $G$.

For $H$ a subgroup of a finite group $G$, the hypernormalizer of $H$ in $G, N_{G}^{\infty}(H)$, is the subgroup in which the ascending chain $H=H_{0} \triangleleft H_{1} \triangleleft H_{2} \triangleleft \cdots$, defined by $H_{i}=N_{G}\left(H_{i-1}\right)$, terminates.

THEOREM 3.2. If the subcofactor $\operatorname{scof}_{G} H=H /$ scor $_{G} H$ of each proper self-normalizing subgroup $H$ of $G$ is p-nilpotent, then there exists a normal p-subgroup $P_{0}$ (which may be trivial) such that $G / P_{0}$ is p-nilpotent. In particular, $G$ is p-solvable of $p$-length $\leqq 2$.

Proof. We use induction on $|G|$. We may assume that $G$ is not $p$-nilpotent, since the result is trivially true otherwise. It suffices to show that $G$ has a nontrivial normal $p$-subgroup $P_{0}$. For then, using Lemma 1.3, we see that the hypotheses hold for $\bar{G}=G / P_{0}$; hence, by induction, $\bar{G}$ has a normal $p$-subgroup $\bar{P}_{1}=P_{1} / P_{0}$ such that $\bar{G} / \bar{P}_{1}$ is $p$-nilpotent so that $P_{1}$ is a normal $p$-subgroup of $G$ and $G / P_{1} \simeq \bar{G} / \bar{P}_{1}$ is $p$-nilpotent.

If $G$ is non- $p$-nilpotent, then by Theorem 3-D, $G$ has a nontrivial $p$-subgroup $P$ and a $p^{\prime}$-element $x$ in $N_{G}(P)$ which does not centralize $P$. Let $N=N_{G}^{\infty}(P)$ be the hypernormalizer of $P$ in $G$. If $N=G$, then $P \triangleleft \triangleleft G$ so that from Lemma 3.1, $G$ has a nontrivial normal $p$-subgroup and the result follows. 
Thus suppose $N \neq G$. Then, by hypothesis, since $N$ is self-normalizing in $G$, $\bar{N}=N / \operatorname{scor}_{G} N$ is $p$-nilpotent. Let $\bar{P}=P\left(\operatorname{scor}_{G} N\right) / \operatorname{scor}_{G} N$ and $\bar{x}=x\left(\operatorname{scor}_{G} N\right)$. Since $x$ normalizes $P, \bar{P} \triangleleft\langle\bar{P}, \bar{x}\rangle$; and since $\langle\bar{P}, \bar{x}\rangle$ is $p$-nilpotent as a subgroup of $\bar{N}$, $\langle\bar{x}\rangle \triangleleft\langle\bar{P}, \bar{x}\rangle$. Since $x$ is a $p^{\prime}$-element, it follows that $\langle\bar{P}, \bar{x}\rangle=\bar{P} \times\langle\bar{x}\rangle$, hence that $\bar{x}$ centralizes $\bar{P}$.

Since $x \notin C_{G}(P)$, there exists $u \in P$ such that $[u, x]=u^{-1} x^{-1} u x \neq 1$. And since $\bar{x}$ centralizes $\bar{P},[u, x] \in \operatorname{scor}_{G} N$; also, $[u, x] \in P$ since $x$ normalizes $P$. Thus $1 \neq[u, x]$ $\in P \cap \operatorname{scor}_{G} N$; and since $P \triangleleft \triangleleft N$, we have $P \cap \operatorname{scor}_{G} N \triangleleft \triangleleft \operatorname{scor}_{G} N \triangleleft \triangleleft G$. Hence, $P \cap \operatorname{scor}_{G} N$ is a nontrivial subnormal $p$-subgroup of $G$ so that from Lemma 3.1, $G$ has a nontrivial normal $p$-subgroup, as we wished to show.

That $G$ is $p$-solvable of $p$-length $\leqq 2$ is immediate.

The Thompson subgroup $J(P)$ of a $p$-group $P$ is defined as in [7] by $J(P)=$ $\langle A \mid A \in \mathscr{A}(P)\rangle$, where $\mathscr{A}(P)$ is the collection of all abelian subgroups of $P$ of maximal order. We will use the fact that $Z(P) \subseteq Z(J(P))$ and the GlaubermannThompson Theorem (see [7, p. 280, Theorem 3.1]):

THEOREM 3-E. Let $P$ be a p-Sylow subgroup of the finite group $G$ with $p$ odd. If $N_{G}(Z(J(P)))$ is p-nilpotent, then so also is $G$.

THEOREM 3.3. If the subcofactor $\operatorname{scof}_{G} H=H / \operatorname{scor}_{G} H$ of each proper abnormal subgroup $H$ of the finite group $G$ is p-nilpotent and either $p$ is odd or the p-Sylow subgroups of $G$ are abelian, then the conclusion of Theorem 3.2 holds.

Proof. As in the proof of Theorem 3.2, if $G$ has a nontrivial normal $p$-subgroup, the result follows by induction. So suppose $G$ has no such subgroup, and thus by Lemma 3.1, no nontrivial subnormal $p$-subgroup; we must show that $G$ is $p$ nilpotent.

Let $P$ be a $p$-Sylow subgroup of $G$ and consider first the case that $P$ is abelian. Then $N=N_{G}(P)$ is a proper abnormal subgroup of $G$, since $P \Varangle G$, so that by hypothesis, $N /$ scor $_{G} N$ has a normal $p$-complement $T /$ scor $_{G} N$. Since $P \triangleleft N, P \cap$ $\operatorname{scor}_{G} N \triangleleft \operatorname{scor}_{G} N \triangleleft \triangleleft G$. And since $G$ has no nontrivial subnormal $p$-subgroup, this means that $P \cap \operatorname{scor}_{G} N=1$, hence that $\operatorname{scor}_{G} N$ is a $p^{\prime}$-group, so $T$ is a normal $p$ complement of $N$. It follows that $N=P \times T$, so $T \subseteq C_{G}(P)$. Since $P$ is abelian, we have $N=P \times T \subseteq C_{G}(P)$, that is, $P \subseteq Z(N)$. Therefore, by Theorem 3-D, $G$ is $p$-nilpotent.

Now consider the case that $p$ is odd and let $\tilde{N}=N_{G}(Z(J(P)))$, where $J(P)$ is the Thompson subgroup of $P$. Since $Z(J(P))$ is characteristic in $P, Z(J(P)) \triangleleft N_{G}(P)$ so that $N_{G}(P) \subseteq \tilde{N}$. Now $\tilde{N} \rtimes G$ since $N_{G}(P) \rtimes G$, and $\tilde{N} \neq G$ since $G$ has no nontrivial subnormal $p$-subgroups. Thus, $\tilde{N} /$ scor $_{G} \tilde{N}$ is $p$-nilpotent by hypothesis.

Suppose $P_{1}=P \cap \operatorname{scor}_{G} \tilde{N} \neq 1$. Then $P_{1} \triangleleft P$ since $\operatorname{scor}_{G} \tilde{N} \triangleleft \tilde{N}$, hence $P_{1} \cap Z(P)$ $\neq 1$. Since $Z(P) \subseteq Z(J(P)), P_{2}=P_{1} \cap Z(J(P)) \neq 1$. Now $P_{2}=Z(J(P)) \cap \operatorname{scor}_{G} \tilde{N}$ $\triangleleft$ scor $_{G} \tilde{N} \triangleleft \triangleleft G$, since $Z(J(P)) \triangleleft \tilde{N}$, so that $P_{2}$ is a nontrivial subnormal $p$-subgroup of $G$, a contradiction. Consequently, $P \cap \operatorname{scor}_{G} \tilde{N}=1$, that is, $\operatorname{scor}_{G} \tilde{N}$ is a $p^{\prime}$-group. From the $p$-nilpotence of $\tilde{N} / \operatorname{scor}_{G} \tilde{N}$, it follows as in the preceding case that $\tilde{N}$ is $p$-nilpotent, and thus, by the Glaubermann-Thompson Theorem, $G$ is $p$-nilpotent. 
We have the following special cases of Theorems 3.2 and 3.3:

Corollary 3.4. If (a) $H /$ scor $_{G} H$ is 2-nilpotent for each self-normalizing $H<G$, or (b) $H /$ scor $_{G} H$ is 2-nilpotent for each abnormal $H<G$ and the 2-Sylow subgroups of $G$ are abelian, then in either case, $G$ is solvable and there exist normal subgroups $H, K$ of $G$ such that $H / K$ is isomorphic to a 2-complement of $G$.

Proof. From the theorems, there exists a normal 2-subgroup $K$ of $G$ such that $G / K$ has a normal 2-complement $H / K$, which is then isomorphic to a 2-complement of $G$ since $K$ is a 2-group. Since $H / K$ has odd order, it is solvable by the FeitThompson Theorem; hence, $G$ is solvable since $K$ and $G / H$ are 2-groups.

Example 2.5 shows that a non-p-nilpotent group having the cofactors of all its subgroups $p$-nilpotent need not have a normal $p$-Sylow subgroup. This is no longer the case, however, if we require that the proper normal subgroups also be $p$ nilpotent. Before establishing this, we first prove the following result (already used in the proof of Theorem 2.6).

THEOREM 3.5. Let $G$ be a solvable non-p-nilpotent group having all proper normal p-subgroups p-nilpotent, and let $|G|=p^{a} m$ with $(p, m)=1$. If $G$ has an abelian normal p-Sylow subgroup $P$, then $G$ has exactly $p^{a}$ distinct $p$-complements.

Proof. Extend $G \supset P \supset 1$ to a chief series $G=G_{0} \supset \ldots \supset G_{m}=P \supset G_{m+1} \supset \ldots \supset$ $G_{m+n}=1$, and set $P_{i}=G_{m+i}$ for $0 \leqq i \leqq n$. Then for each $i \geqq 1, G / P_{i}$ is not $p$-nilpotent. For if $K / P_{i}$ were a normal $p$-complement of $G / P_{i}$ for some $i \geqq 1$, then since $p|| G / P_{i} \mid$, $K$ is a proper normal subgroup of $G$, hence has a characteristic $p$-complement $T$ which is then easily seen to be a normal $p$-complement of $G$, making $G$ p-nilpotent.

Also, if $\left(G / P_{i}\right)_{(p)}^{\prime}$ is the smallest normal subgroup of $G / P_{i}$ for which the factor group is an abelian $p$-group, then for each $i \geqq 1,\left(G / P_{i}\right)_{(p)}^{\prime}=G / P_{i}$. For if $\left(G / P_{i}\right)_{(p)}^{\prime}=L / P_{i}$ were properly contained in $G / P_{i}$, then $L$, being a proper normal subgroup of $G$, would be $p$-nilpotent. But then $L / P_{i}$ would also be $p$-nilpotent and its characteristic $p$-complement would then be a normal $p$-complement of $G / P_{i}$, contradicting what we have just shown.

Now for each $i \geqq 1$, let $\tau_{i}$ be the transfer of $G / P_{i}$ into its abelian normal $p$-Sylow subgroup $P / P_{i}$. Then $\operatorname{ker}\left(\tau_{i}\right)=\left(G / P_{i}\right)_{(p)}^{\prime}$, and $\tau_{i}\left(G / P_{i}\right)=\left(P / P_{i}\right) \cap Z\left(G / P_{i}\right)$. It follows from what we have just shown that $\left(P / P_{i}\right) \cap Z\left(G / P_{i}\right)=1$ for all $i \geqq 1$.

For each $i \geqq 1$, therefore, since $P_{i-1} \subseteq P$, we have $\left(P_{i-1} / P_{i}\right) \cap Z\left(G / P_{i}\right)=1$, which means that each of $P / P_{1}, P_{1} / P_{2}, \ldots, P_{n-1} / P_{n}$ is an eccentric chief factor of $G$. A result of $P$. Hall [8] states that, for a solvable group $G$, the number of $p$-complements of $G$ is equal to the product of the orders of the eccentric $p$-chief factors of $G$. Thus the number of $p$-complements of $G$ is $\prod_{i=1}^{n}\left|P_{i-1} / P_{i}\right|=|P|=p^{a}$.

Although Example 2.8 shows that adding to the hypotheses of Theorems 3.2 or 3.3 the condition that the proper normal subgroups be $p$-nilpotent is not enough to give Itô's result, we do find some additional structure in $G$. 
THEOREM 3.5. Let $G$ be a non-p-nilpotent group having all its proper normal subgroups p-nilpotent, and let $|G|=p^{a} m$ with $(p, m)=1$. Suppose also that the hypothesis of Theorem 3.2 or of Theorem 3.3 holds. Then

(1) $G$ has a normal p-Sylow subgroup $P$.

(2) $P \subseteq G^{\prime}$.

(3) $F_{p}(G)=$ the largest normal p-nilpotent subgroup of $G$ is the unique maximal normal subgroup of $G$ and $G / F_{p}(G)$ is a $p^{\prime}$-group.

(4) For all proper normal subgroups $K$ of $G,\left([G: K], d_{p}\right) \neq 1$ where

$$
d_{p}=\prod_{i=1}^{a}\left(p^{i}-1\right)
$$

(5) If $P$ is abelian, then $C_{G}(P)=F_{p}(G)$, and thus $G$ induces a $p^{\prime}$-group of automorphisms in $P$.

(6) If $G$ is solvable, then $\left[G: F_{p}(G)\right]=q$ for some prime $q \in \pi(G)$ dividing $d_{p}$; if also $P$ is abelian, then $G$ has exactly $p^{a}$ distinct p-complements.

Proof. Most of the conclusions are immediate. For (2), the proof of (6) of Theorem 2.6 carries over with $p_{i}=p$. (3) is trivial, since every. proper normal subgroup of $G$ must be contained in $F_{p}(G)$; in particular, $P \subseteq F_{p}(G)$. (6) will follow from the fact that, in a solvable group, maximal normal subgroups have prime index, together with (4) and Theorem 3.4.

(1) From Theorems 3.2 and 3.3, $G$ has a normal $p$-subgroup $P_{0}$ such that $G / P_{0}$ has a normal $p$-complement $K / P_{0}$. If $P_{0}$ is not a $p$-Sylow subgroup of $G$, then $K$ would be a proper normal subgroup of $G$, hence $p$-nilpotent. But it is easily seen that its characteristic $p$-complement would be a normal $p$-complement of $G$, contradicting the non-p-nilpotence of $G$. Thus, $G$ has a normal $p$-Sylow subgroup.

(4) Let $G$ be a minimal counterexample. Then there exists $L_{\ddagger}^{\triangleleft} G$ with ([G:L], $d_{p}$ ) $=1$. Let $F=F_{p}(G)$. Then $L \subseteq F$ from (3) so that $[G: F]=n$ also is prime to $d_{p}$ as well as to $p$. Suppose now that $P$ is not minimal normal in $G$, and let the normal subgroup $M \neq 1$ of $G$ be properly contained in $P$. If $G / M$ were $p$-nilpotent, say with normal $p$-complement $U / M$, then $U$ would be a proper normal subgroup of $G$, hence $p$-nilpotent, and its characteristic $p$-complement would be a normal $p$ complement of $G$. Thus, $G / M$ is not $p$-nilpotent; and using Lemma 1.3, we see that the other hypotheses hold for $G / M$. But $F / M$ is a proper normal subgroup of $G / M$ and $[G / M: F / M]=[G: F]=n$ is prime to $d_{p}$, hence is prime to $d_{p}^{*}=\prod_{i=1}^{s}\left(p^{i}-1\right)$, where $p^{s}$ is the $p$-part of $|G / M|$. This means that $G / M$ is a counterexample of order less than $|G|$, contradicting the minimality of $G$. Therefore, $P$ is minimal normal n $G$, hence is elementary abelian so that $\mid$ Aut $(P) \mid=p^{e} \cdot d_{p}$, where $e=a(a-1) / 2$.

Now, since $P$ is abelian and $P \subseteq F$ which is $p$-nilpotent, both $P$ and the normal $p$-complement $T$ of $F$ centralize $P$; thus $F=P \times T \subseteq C_{G}(P)$, from which it follows that $\left|G / C_{G}(P)\right|$ divides $[G: F]=n$, and so is prime to both $p$ and $d_{p}$. However, $\left|G / C_{G}(P)\right|$ must divide $\mid$ Aut $(P) \mid=p^{e} \cdot d_{p}$. Thus we have a contradiction, so no such minimal counterexample can exist. 
(5) From the proof of (4), $F_{p}(G) \subseteq C_{G}(P)$; and from Theorem 3-D, $P \nsubseteq Z\left(N_{G}(P)\right)$ $=Z(G)$. Thus, by the maximality of $F_{p}(G)$, we have $F_{p}(G)=C_{G}(P)$.

If we call a subgroup $H$ of $G$ somewhat normal in $G$ if $\operatorname{cof}_{G} H=H / \operatorname{cor}_{G} H$ is cyclic of prime-power order, and extend the requirement of $p$-nilpotence from the proper normal subgroups of $G$ to the proper somewhat normal subgroups, then we recover all of Itô's result.

THEOREM 3.6. Let $G$ be a finite non-p-nilpotent group having all its proper somewhat normal subgroups p-nilpotent. Suppose also that the hypothesis of Theorems 3.2 or 3.3 holds. Then

(i) $|G|=p^{a} q^{b}$ for some prime $q \neq p$; in particular, $G$ is solvable.

(ii) All proper subgroups of $G$ are nilpotent.

(iii) The conclusions of Theorems 3-A and 3.5 hold.

Proof. (i) From Theorem 3.5, $G$ has a normal $p$-Sylow subgroup $P$, and from Theorem 3-D, $G$ has an element $x$ of order $q^{b}$ for some prime $q \neq p$, where $x \notin C_{G}(P)$. Now $P \subseteq \operatorname{cor}_{G}(P\langle x\rangle)$, so $P\langle x\rangle / \operatorname{cor}_{G}(P\langle x\rangle)$ is a homomorphic image of $P\langle x\rangle / P$ $\simeq\langle x\rangle$, hence is cyclic of prime-power order. Thus $P\langle x\rangle$ is a somewhat normal subgroup of $G$. If $P\langle x\rangle \neq G$, then by hypothesis, $P\langle x\rangle$ is $p$-nilpotent, from which it follows that $P\langle x\rangle=P \times\langle x\rangle$. But this implies that $x$ centralizes $P$, a contradiction. Therefore, $G=P\langle x\rangle$, so $|G|=p^{a} q^{b}$. The solvability of $G$ now follows from the well-known theorem of Burnside.

(ii) Since $P \triangleleft G$ and $|G|=p^{a} q^{b}, G$ is $q$-nilpotent, and thus so also are all its subgroups. In particular, the unique maximal normal subgroup $F=F_{p}(G)$ is both $p$ - and $q$-nilpotent, hence is nilpotent. Now let $S$ be any maximal subgroup of $G$, so $S$ is $q$-nilpotent. Either $S=F$, in which case $S$ is nilpotent, or $S F=G$. In this last case, since $F$ is nilpotent, $S \cap F \triangleleft \triangleleft F \triangleleft G$ so that $S \cap F \subseteq \operatorname{scor}_{G} S=\operatorname{cor}_{G} S$; also, $|S \cap F|=|S||F| /|G|=|S| / q$. Hence, $S / \operatorname{cor}_{G} S$ has order 1 or $q$, so $S$ is somewhat normal (in fact, nearly normal) in $G$. By hypothesis, therefore, $S$ is $p$-nilpotent, and thus is nilpotent. This means that all maximal subgroups (and hence all proper subgroups) of $G$ are nilpotent.

(iii) now follows immediately from (ii).

We conclude this section with one result in which we again strengthen the conditions imposed in Theorem 3.4. Since the $p$-nilpotence of a subgroup or of the subcofactor of a subgroup provides no useful information in case it has order prime to $p$, we might hope to obtain more structure in $G$ if we impose some additional condition on these. Although Example 2.8 shows that the conditions of the following theorem are not sufficient to give Itô's result, we do obtain some additional information about $G$.

We will call a finite group $G(p: q)$-nilpotent if (i) $G$ is $p$-nilpotent; (ii) $q|| G \mid$ and $G$ is $q$-nilpotent in case $p \nmid|G|$ and $|G|>1$.

THEOREM 3.7. Let $G$ be a finite group and $p$ a prime factor of $|G|$ for which every proper normal subgroup $K$ is $\left(p: q_{K}\right)$-nilpotent for some prime $q_{K}$ depending on $K$. 
Suppose also that the cofactor $H / \operatorname{cor}_{G} H$ of each proper subgroup $H$ of $G$ is $\left(p: q_{H}\right)$ nilpotent for some prime $q_{H}$ depending on $H$. Then

(i) $G$ is solvable.

(ii) $G$ has a normal Sylow subgroup.

(iii) If $G$ is not p-nilpotent, then the conclusions of Theorem 3.4 hold; in particular, $G$ has a normal p-Sylow subgroup, and if $P$ is abelian, then $G$ has exactly $p^{a}$ distinct p-complements where $p^{a}$ is the p-part of $|G|$.

Proof. Case 1. $G$ is $p$-nilpotent.

Let $|G|=\prod_{i=1}^{r} p_{i}^{a_{i}}$, where $p_{1}=p$ and the $p_{i}$ are distinct primes dividing $|G|$. We may assume $r>1$, since the result is trivially true otherwise. Then there exists $T_{1} \triangleleft G$ with $\left[G: T_{1}\right]=p_{1}^{a_{1}}$, since $G$ is $p_{1}$-nilpotent. Since $T_{1}$ is a proper nontrivial normal $p_{1}^{\prime}$-subgroup, it is $p_{i}$-nilpotent for some $i \geqq 2$, say for $i=2$. Thus there exists $T_{2}$ characteristic in $T_{1}$, hence normal in $G$, with $\left[T_{1}: T_{2}\right]=p_{2}^{a_{2}}$. Continuing gives a normal series of $G, G=T_{0} \supset T_{1} \supset T_{2} \supset \ldots \supset T_{r}=1$, where for each $i \geqq 1$, $\left|T_{i-1} / T_{i}\right|=p_{i}^{a_{i}}$. It follows that $G$ is solvable with a normal $p_{r}$-Sylow subgroup $T_{r-1}$.

Case 2. $G$ is not p-nilpotent.

Then, by Theorem 3.4, $G$ has a normal $p$-Sylow subgroup $P$ so that (ii) holds. We consider two subcases.

(a) Suppose $P$ is not minimal normal in $G$. Let $M \neq 1$ be a normal subgroup of $G$ properly contained in $P$. We show that the hypotheses hold for $G / M$. If $H / M<G / M$, then $\operatorname{cof}_{G} H=H / \operatorname{cor}_{G} H$ is $\left(p: q_{H}\right)$-nilpotent for some prime $q_{H}$, and thus $\operatorname{cof}_{G / M}(H / M) \simeq \operatorname{cof}_{G} H$ also is $\left(p: q_{H}\right)$-nilpotent relative to this same prime $q_{H}$. Clearly all proper normal subgroups of $G / M$ are $p$-nilpotent. Now suppose $K / M$ is a proper nontrivial normal $p^{\prime}$-subgroup of $G / M$. Then $K$ is $p$-nilpotent by hypothesis, say with characteristic $p$-complement $T$. Then $T$ is normal in $G$, and since $M$ is the $p$-Sylow subgroup of $K, K=M \times T$. Since $T \neq 1$ is a $p^{\prime}$-subgroup, it is $q$ nilpotent for some prime $q$ dividing $|T|$. Hence, since $K / M \simeq T, q$ divides $|K| M \mid$ and $K / M$ is $q$-nilpotent.

The hypotheses thus hold for $G / M$, so by induction, $G / M$ is solvable. Since $M$ is a $p$-group, $G$ also is solvable.

(b) Suppose $P$ is minimal normal in $G$. Then either there is a minimal normal subgroup $L$ of $G$ distinct from $P$, or $P$ is the unique minimal normal subgroup of $G$.

In the first case, $L \cap P=1$ so that $L$ is a proper normal $p^{\prime}$-subgroup of $G$, hence is $q$-nilpotent for some prime $q$ dividing $|L|$; and since the normal $q$-complement is characteristic in $L$, it follows from the minimality of $L$ that $L$ is a $q$-group. As in (a), the conditions on the cofactors of subgroups of $G / L$ are satisfied, and all proper normal subgroups of $G / L$ are $p$-nilpotent. Now let $K / L$ be a nontrivial proper normal $p^{\prime}$-subgroup of $G / L$. Then by hypothesis, $K$ is $q_{1}$-nilpotent for some prime $q_{1}$ dividing $|K|$, say with normal $q_{1}$-complement $U$. Now, if $q_{1}|| K / L \mid$, we have exhibited a prime $q_{1}$ dividing $|K / L|$ for which $K / L$ is $q_{1}$-nilpotent. So suppose that $q_{1}$ does not divide $|K / L|$. This means that $q_{1}=q$ so that $K=U \times L$. Now $U$ is a 
nontrivial proper normal $p^{\prime}$-subgroup of $G$, so it is $q_{2}$-nilpotent for some prime $q_{2}$ dividing $|U|$. Since $K / L \simeq U$, we have that $q_{2}|| K / L \mid$ and that $K / L$ is $q_{2}$-nilpotent. We have thus shown that the hypotheses hold for $G / L$; by induction, therefore, $G / L$ is solvable, and hence, so is $G$.

There remains to consider only the possibility that $P$ is the unique minimal normal subgroup of $G$. By the Schur-Zassenhaus Theorem, $P$ has a complement $T$ in $G$. Then if $H$ is any subgroup of $T$ (not necessarily proper), $H$ is a proper $p^{\prime}$ subgroup of $G$; and by the uniqueness of $P$, we have $\operatorname{cor}_{G} H=1$ so that $H=\operatorname{cof}_{G} H$. By hypothesis, therefore, each nontrivial subgroup $H$ of $T$, including $T$ itself, is $q_{H}$-nilpotent for some prime $q_{H}$ dividing $|H|$ (where $q_{H}$ depends on $H$ ). Arguing as in Case 1 with $G$ replaced by $T$, we find that $T$ is solvable. Thus $G / P \simeq T$ is solvable, from which it follows that $G$ is solvable.

(iii) is immediate.

4. $\theta=$ supersolvable or $\sigma$-Sylow-towered. In this section we examine the influence on a group $G$ of supersolvable subcofactors of certain subgroups of $G$, and more generally, of $\sigma$-Sylow-towered subcofactors. A finite group $G$ is said to have a $\sigma$-Sylow-tower, where $\sigma=\left(p_{1}, \ldots, p_{m}\right)$ is some ordering of a set $\Sigma=\left\{p_{1}, \ldots, p_{m}\right\}$ of primes containing $\pi(G)$, if there is a normal series of $G, 1=K_{0} \subseteq K_{1} \subseteq \cdots \subseteq K_{m}$ $=G$, such that for each $i=1, \ldots, m, K_{i} / K_{i-1}$ is isomorphic to a $p_{i}$-Sylow subgroup of $G$ (which may be trivial in case $p \nmid|G|$ ). For example, if $\sigma$ is the natural descending order of $\Sigma$, then it is well known (see [7, p. 716, Satz 9.1]) that if $G$ is supersolvable, then $G$ has a $\sigma$-Sylow-tower.

The classic result in this direction is the following well-known theorem of Huppert [9]:

THEOREM 4-A. If all the proper subgroups of the finite group $G$ are supersolvable, then $G$ is solvable.

Doerk in [5] has extended this result by describing much of the structure of $G$. Several of his results parallel those of the Schmidt-Iwasawa Theorem given in \$3. The following are extensions due to Rose [13], [14]:

THEOREM 4-B. Let $G$ be a finite group and $\sigma$ a fixed ordering of a set $\Sigma$ of primes containing $\pi(G)$.

(i) If (a) every proper self-normalizing subgroup $H$ of $G$ has a $\sigma$-Sylow-tower, or if (b) every proper abnormal subgroup $H$ of $G$ has a $\sigma$-Sylow-tower and the 2-Sylow subgroups of $G$ are abelian, then in either case, $G$ is solvable.

(ii) If every proper abnormal subgroup $H$ of $G$ is supersolvable and the abnormal maximal subgroups of $G$ have prime-power index, then $G$ is solvable.

[Example 3-C shows the need for the additional condition in (ii) and in (i(b)).]

We will now show that it is sufficient to impose the conditions in these results of Rose on only the subcofactors $H /$ scor $_{G} H$. First, however, we establish a preliminary lemma and theorem. 
Lemma 4.1. For $G$ a group, $F_{2}\left(G^{\prime}\right)=G^{\prime}$ (that is, $G^{\prime}$ has Fitting length $\leqq 2$ ) if and only if $G / F_{2}(G)$ is abelian.

Proof. Using the equality $F\left(G^{\prime}\right)=F(G) \cap G^{\prime}$ and the normality of $G^{\prime}$ and $F(G)$, we have the following chain of equivalences: $F_{2}\left(G^{\prime}\right)=G^{\prime} \leftrightarrow G^{\prime} \mid F\left(G^{\prime}\right)$ is nilpotent $\leftrightarrow G^{\prime} \mid F(G) \cap G^{\prime}$ is nilpotent $\leftrightarrow G^{\prime} F(G) / F(G)$ is nilpotent $\leftrightarrow G^{\prime} F(G) / F(G)$ is contained in $F(G / F(G))=F_{2}(G) / F(G) \leftrightarrow G^{\prime} \subseteq F_{2}(G) \leftrightarrow G / F_{2}(G)$ is abelian.

THEOREM 4.2. Let $G$ be a finite solvable group for which the cofactors of the maximal subgroups are supersolvable. Then

(i) $G / F(G)$ is supersolvable.

(ii) $f\left(G^{\prime}\right) \leqq 2$, that is, $F_{2}\left(G^{\prime}\right)=G^{\prime}$, or equivalently, $G / F_{2}(G)$ is abelian.

(iii) $f(G) \leqq 3$, that is, $F_{3}(G)=G$.

Proof. (i) follows immediately from Corollary 1.4(ii). For (ii), since $G / F(G)$ is supersolvable, its derived subgroup $(G / F(G))^{\prime}=G^{\prime} F(G) / F(G)$ is nilpotent, hence so is the isomorphic group $G^{\prime} / G^{\prime} \cap F(G)$, which is in turn equal to $G^{\prime} / F\left(G^{\prime}\right)$. Therefore, $G^{\prime} / F\left(G^{\prime}\right)$ is nilpotent so that $F_{2}\left(G^{\prime}\right)=G^{\prime}$. (iii) follows from (ii).

That $m=2$ and $n=3$ are the best possible integers for which $G^{\prime}=F_{m}\left(G^{\prime}\right)$ and $G=F_{n}(G)$ in the preceding theorem is seen by taking $G=S_{4} . S_{4}$ is solvable, the cofactors of all proper subgroups of $S_{4}$ are supersolvable, the Fitting length of $S_{4}^{\prime}=A_{4}$ is 2 , and the Fitting length of $S_{4}$ is 3 .

Example 3-C shows that the solvability of $G$ is needed in Theorem 4.2, that the supersolvability of the cofactors of the maximal subgroups of $G$ is not sufficient to guarantee that $G$ is solvable. The following extension of (a(i)) of Theorem 4-B shows, however, that if we enlarge the class of subgroups which are to have supersolvable cofactors (and more generally, $\sigma$-Sylow-towered subcofactors) to the collection of all self-normalizing subgroups of $G$, then $G$ is solvable.

THEOREM 4.3. Let $\sigma$ be a fixed ordering of a set $\Sigma$ of primes containing $\pi(G)$. If the subcofactor $\mathrm{H} / \mathrm{scor}_{G} H$ of each proper self-normalizing subgroup $H$ of $G$ has $a$ $\sigma$-Sylow-tower, then $G$ is solvable. Moreover, $G / F(G)$ has a $\sigma$-Sylow-tower.

Proof. We first show that $G$ is solvable, using induction on $|G|$. If $G$ is simple, the solvability follows from Theorem 4-B, since in this case, every proper subgroup has subnormal core $=1$ so that each proper self-normalizing subgroup is $\sigma$-Sylowtowered. Thus suppose $G$ is not simple, and let $M$ be a minimal normal subgroup of $G$. Since $\pi(G / M) \subseteq \pi(G) \subseteq \Sigma$ and the subcofactors of self-normalizing subgroups of $G / M$ are $\sigma$-Sylow-towered by Lemma $1.3, G / M$ is solvable by induction.

We show now that the hypotheses hold for $M$. If $H$ is any self-normalizing (in $M$ ) subgroup of $M$, then $N=N_{G}^{\infty}(H)=$ the hypernormalizer of $H$ in $G$ is a selfnormalizing subgroup of $G$. $M \nsubseteq N$, for otherwise $H \triangleleft \triangleleft M$ since $H \triangleleft \triangleleft N$. Thus $N \neq G$ so that $N /$ scor $_{G} N$ is $\sigma$-Sylow-towered by hypothesis, and hence so also is its subgroup $H\left(\operatorname{scor}_{G} N\right) / \operatorname{scor}_{G} N$. Since $H \triangleleft \triangleleft N, H \cap \operatorname{scor}_{G} N \triangleleft \triangleleft \operatorname{scor}_{G} N \triangleleft \triangleleft G$, so $H \cap \operatorname{scor}_{G} N$ is contained in $\operatorname{scor}_{G} H$, which in turn is easily seen to be contained 
in $\operatorname{scor}_{M} H$. Therefore, $H /$ scor $_{M} H$, being a homomorphic image of $H / H \cap \operatorname{scor}_{G} N$ $\simeq H\left(\operatorname{scor}_{G} N\right) /$ scor $_{G} N$ is $\sigma$-Sylow-towered. Since $\pi(M) \subseteq \pi(G) \subseteq \Sigma$, the hypotheses hold for $M$ so that by induction, $M$ is solvable; and the solvability of $G$ now follows.

The fact that $G / F(G)$ has a $\sigma$-Sylow-tower is an immediate consequence of Corollary 1.4(ii), since the property of being $\sigma$-Sylow-towered is easily seen to be a strictly homomorphism-invariant property.

We also have the following extension of (i(b)) of Theorem 4-B:

THEOREM 4.4. Let $\sigma=\left(p_{1}, \ldots, p_{n}\right)$ be a fixed ordering of the set $\left\{p_{1}, \ldots, p_{n}\right\}$ of primes containing $\pi(G)$. If the subcofactor $H / \operatorname{scor}_{G} H$ of each proper abnormal subgroup $H$ of $G$ has a $\sigma$-Sylow-tower and the 2-Sylow subgroups of $G$ are abelian, then $G$ is solvable, and $G / F(G)$ has a $\sigma$-Sylow-tower.

Proof. The last part follows as in the proof of Theorem 4.3, so only the solvability of $G$ requires proof. For this, suppose $G$ is a minimal counterexample. If $G$ has a nontrivial solvable normal subgroup $K$, then by the minimality of $G, G / K$ would be solvable and the solvability of $G$ would follow. Thus, $G$ has no nontrivial normal solvable subgroup, and so, from Lemma 3.1, no nontrivial subnormal solvable subgroup.

Now consider a maximal segment of a $\sigma$-Sylow-tower for $G$, that is, a normal chain $K_{r} \subseteq K_{r+1} \subseteq \cdots \subseteq K_{n}=G$ such that there is no $G$-normal subgroup $H$ of $K_{r}$ for which $K_{r} / H$ is isomorphic to a $p_{r}$-Sylow subgroup of $G$, and in case $r<n$. $K_{i} / K_{i-1}$ is isomorphic to a $p_{i}$-Sylow subgroup of $G$ for each $i=r+1, \ldots, n$. The first condition is clearly equivalent to saying that $K_{r}$ is not $p_{r}$-nilpotent. Since $G$ is not solvable, hence not $\sigma$-Sylow-towered, we have $r>0$ so that $K_{r} \neq 1$.

Case 1. $p_{r}=2$.

Let $P$ be a 2-Sylow subgroup of $K=K_{r}$, and thus a 2-Sylow subgroup of $G$ since $2 \nmid[G: K]$. Then $N=N_{G}(P) \rtimes G$ and $N \neq G$ since $G$ has no nontrivial normal solvable subgroups. By hypothesis, therefore, $N / \operatorname{scor}_{G} N$ has a $\sigma$-Sylow-tower. Since $P \triangleleft N, P \cap \operatorname{scor}_{G} N \triangleleft \operatorname{scor}_{G} N \triangleleft \triangleleft G$ so that $P \cap \operatorname{scor}_{G} N=1$ since $G$ has no nontrivial subnormal solvable subgroups. This means that $\operatorname{scor}_{G} N$ has odd order, hence is solvable by the Feit-Thompson Theorem, and thus $\operatorname{scor}_{G} N=1$ since $G$ has no subnormal solvable subgroups $\neq 1$. Consequently, $N=N / \operatorname{scor}_{G} N$ has a $\sigma$-Sylow-tower, and thus so also does its subgroup $N_{K}(P)=N \cap K$.

Since $p_{r+1}, \ldots, p_{n}$ do not divide $|K|$, it follows that $N_{K}(P)$ has a $\left(p_{1}, \ldots, p_{r}\right)$ Sylow-tower, in particular, $N_{K}(P)$ has a normal $p_{r}$-complement $T$. And since $P$ and $T$ are normal in $N_{K}(P), N_{K}(P)=P \times T$ so that $T$ centralizes $P$. But $P$ is abelian by hypothesis, so $P \subseteq C_{G}(P)$ also. It follows that $P \subseteq Z\left(N_{K}(P)\right)$, and hence from Theorem 3-E, that $K=K_{r}$ is $p_{r}$-nilpotent, a contradiction.

Case 2. $p_{r}$ is odd.

As before, let $P$ be a $p_{r}$-Sylow subgroup of $K=K_{r}$ and thus a $p_{r}$-Sylow subgroup of $G$. Let $J(P)=$ the Thompson subgroup of $P$, and $\tilde{N}=N_{G}(Z(J(P)))$. Now $Z(J(P))$ 
$\triangleleft N_{G}(P)$ so $N_{G}(P) \subseteq \tilde{N}$, and since $N_{G}(P) \rtimes G, \tilde{N}$ is also abnormal in $G$. $\tilde{N} \neq G$ since $G$ has no nontrivial normal solvable subgroups. By hypothesis, therefore, $\tilde{N} / \operatorname{scor}_{G} \tilde{N}$ is $\sigma$-Sylow-towered, so its subgroup $\left(\tilde{N}_{K}\right)\left(\operatorname{scor}_{G} \tilde{N}\right) / \operatorname{scor}_{G} \tilde{N}$ also is, where $\tilde{N}_{K}=N_{K}(Z(J(P)))=\tilde{N} \cap K$. It follows that $\left(\tilde{N}_{K}\right)\left(\operatorname{scor}_{G} \tilde{N}\right) / \operatorname{scor}_{G} \tilde{N}$ is $p_{r}$-nilpotent, as in Case 1 , hence so is $\tilde{N}_{K} / \tilde{N}_{K} \cap \operatorname{scor}_{G} \tilde{N}$.

Arguing as in the proof of Theorem 3.3, we find that if $P_{1}=P \cap \operatorname{scor}_{G} \tilde{N}$ is nontrivial, then $P_{2}=P_{1} \cap Z(J(P))$ is a nontrivial subnormal subgroup of $G$, which contradicts $G$ having no subnormal solvable subgroups $\neq 1$. Thus $P \cap \operatorname{scor}_{G} \tilde{N}=1$ so that $\operatorname{scor}_{G} \tilde{N}$ is a $p_{r}^{\prime}$-group, and hence so also is $\tilde{N}_{K} \cap \operatorname{scor}_{G} \tilde{N}$. Since $\tilde{N}_{K} / \tilde{N}_{K} \cap \operatorname{scor}_{G} \tilde{N}$ is $p_{r}$-nilpotent, this implies that $\tilde{N}_{K}=N_{K}(Z(J(P)))$ also is $p_{r}$-nilpotent. But then by the Glaubermann-Thompson result, we again have $K=K_{r}$ is $p_{r}$-nilpotent, a contradiction.

Each case, therefore, leads to a contradiction so that no such minimal counterexample can exist.

The preceding two results give the following corollary.

Corollary 4.5. If (a) the subcofactor $\mathrm{H} / \mathrm{scor}_{G} \mathrm{H}$ of each proper self-normalizing subgroup $H$ of $G$ is supersolvable, or if (b) the subcofactor $H /$ scor $_{G} H$ of each proper abnormal subgroup $H$ of $G$ is supersolvable and the 2-Sylow subgroups of $G$ are abelian, then in either case, $G$ is solvable. Moreover, $G / F(G)$ is supersolvable and $G / F_{2}(G)$ is abelian; thus, $f\left(G^{\prime}\right) \leqq 2$ and $f(G) \leqq 3$.

Proof. The solvability of $G$ follows from Theorems 4.3 and 4.4 and the comments in the opening paragraph of this section. The last part follows by Theorem 4.2.

We can similarly extend Theorem 4-B(ii). For this we need the following lemma given by Rose in [14].

Lemma 4-C. Suppose $G$ is simple and that $G=H K$ where $H$ and $K$ are proper subgroups of $G$. Then $\operatorname{cor}_{H}(H \cap K)=\operatorname{cor}_{K}(H \cap K)=1$.

Proof. One shows that for $C=\operatorname{cor}_{H}(H \cap K)$ (and similarly for $\operatorname{cor}_{K}(H \cap K)$ ), the normal closure of $C=C^{G} \subseteq K$, so by the simplicity of $G, C^{G}=1$, hence $C=1$.

THEOREM 4.6. If the subcofactor $H /$ scor $_{G} H$ of each proper abnormal subgroup $H$ of $G$ is supersolvable and the abnormal maximal subgroups of $G$ have prime-power index, then $G$ is solvable. Moreover, $G / F(G)$ is supersolvable and $G / F_{2}(G)$ is abelian; thus $f\left(G^{\prime}\right) \leqq 2$ and $f(G) \leqq 3$.

Proof. The last statement follows as before, and only the solvability of $G$ requires proof. For this, we use induction on $|G|$. If $G$ is simple, then all maximal subgroups of $G$ are abnormal in $G$ and have subnormal core=1, so $G$ is solvable by Huppert's theorem or by Theorem 4-B(ii). So suppose $G$ is not simple, and let $M$ be a minimal normal subgroup. By induction, $G / M$ is solvable; and if $G$ has another minimal normal subgroup $M^{*} \neq M$, the solvability of $G=G / M \cap M^{*}$ follows. We may assume, therefore, that $M$ is the unique minimal normal subgroup of $G$, 
and we need only show that $M$ is solvable. We suppose it is not, and work toward a contradiction.

If $M$ is not solvable, then $M=M_{1} \times \cdots \times M_{k}$, where the $M_{i}$ are isomorphic simple nonabelian groups. $M \nsubseteq \Phi(G)$ since $M$ is not solvable, so there exist maximal subgroups of $G$ not containing $M$. By the uniqueness of $M$, we see that $G$ has maximal subgroups of core 1 .

Suppose there is only one conjugacy class $\mathscr{C}$ of such maximal subgroups of core 1. By hypothesis, for some power $p^{a}$ of a prime $p,[G: S]=p^{a}$ for all $S \in \mathscr{C}$. Since $M \nsubseteq S$ for $S \in \mathscr{C}$, we see that $p|| M \mid$. Let $P$ be a $p$-Sylow subgroup of $M$; then $1 \neq P$, and $P \neq M$ since $M$ is not solvable. By the Frattini argument, $G=$ $M N_{G}(P)$; and by the minimality of $M, N_{G}(P) \neq G$ so that $N_{G}(P) \subseteq T$ for some maximal subgroup $T$ of $G$. Since $G=M N_{G}(P)=M T$, we have $M \nsubseteq T$, so $\operatorname{cor}_{G} T=1$ by the uniqueness of $M$. It follows that $T \in \mathscr{C}$ so that $p^{a}=[G: T]=[M T: T]=$

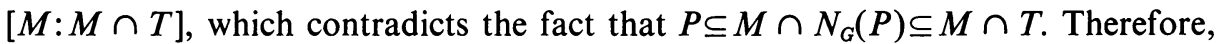
$G$ has at least two distinct conjugacy classes of maximal subgroups of core 1.

Now let $S$ be a maximal subgroup with $\operatorname{cor}_{G} S=\operatorname{scor}_{G} S=1$, and let $P$ be a $p$ Sylow subgroup of $S$ where $p=\max (\pi(S))$. Then $S \rtimes G$ so that $S=S /$ scor $_{G} S$ is supersolvable by hypothesis; in particular, $P \triangleleft S$, hence $S \subseteq N_{G}(P)$. Since $G$ has no nontrivial solvable normal subgroups, it follows that $S=N_{G}(P)$; also, it is now clear that $P$ is a $p$-Sylow subgroup of $G$.

Thus, if $S$ and $T$ are maximal subgroups of $G$ with core 1 and $p=\max (\pi(S))$ $=\max (\pi(T))$, then $S=N_{G}(P)$ and $T=N_{G}\left(P^{*}\right)$ for some $p$-Sylow subgroups $P$ and $P^{*}$ of $G$. Since $P$ and $P^{*}$ are conjugate in $G$, so are $S$ and $T$. Therefore, if $S$ is any maximal subgroup of $G$ with core 1 and $p=\max (\pi(S))$, then the conjugacy class of $S$ is $\mathscr{C}(p)=\left\{N_{G}(P) \mid P\right.$ a $p$-Sylow subgroup of $\left.G\right\}$.

If $\mathscr{C}\left(p_{1}\right)$ and $\mathscr{C}\left(p_{2}\right)$ are two such conjugacy classes with $p_{1}>p_{2}$, then $p_{1}=p$ $=\max (\pi(G))$. For if $T \in \mathscr{C}\left(p_{2}\right)$, then $p_{2}=\max (\pi(T))$; and since $p \geqq p_{1}>p_{2}$, this implies that both $p$ and $p_{1}$ divide [G:T], a contradiction unless $p=p_{1}$.

Now let $S \in \mathscr{C}\left(p_{1}\right)=\mathscr{C}(p)$ and $T \in \mathscr{C}\left(p_{2}\right)$. Then $[G: T]$ is a power of $p$ and $p \nmid|T|$ so that $T$ is a $p$-complement of $G$. Let $q \mid[G: S]$ and let $U$ be a $q$-complement of $S$, hence of $G$. ( $U$ exists since $S=S /$ scor $_{G} S$ is supersolvable, hence solvable.) Then, as Rose shows in [14], $A=T \cap U \cap M$ is an abelian Hall $\{p, q\}^{\prime}$-subgroup of $G$. We include the argument here.

First, since $M \triangleleft G, T \cap M$ and $U \cap M$ are $p$ - and $q$-complements respectively of $M$, so for each $i=1, \ldots, k, T \cap M_{i}$ and $U \cap M_{i}$ are $p$ - and $q$-complements of $M_{i}$. It follows that $M_{i}=\left(T \cap M_{i}\right)\left(U \cap M_{i}\right)$; and since $T \cap M_{i}$ and $U \cap M_{i}$ are supersolvable while $M$ is not, these are proper subgroups of $M_{i}$. Hence, by Lemma 4-C, $A_{i}=T \cap U \cap M_{i}$ contains no nontrivial normal subgroup of either $T \cap M_{i}$ or $U \cap M_{i}$.

Now, by straightforward computation, we see that $A_{i}$ is a Hall $\{p, q\}^{\prime}$-subgroup of $M_{i}$ for each $i$; in particular, $A_{i}$ is a $q$-complement of $T \cap M_{i}$ and a $p$-complement of $U \cap M_{i}$. Also, each $A_{i}$ is abelian. To see this, let $P$ be a $p$-Sylow subgroup of 
$U \cap M_{i}$. Since $p=\max \left(\pi\left(U \cap M_{i}\right)\right)$ and $U \cap M_{i}$ is supersolvable, $P \triangleleft U \cap M_{i}$ so that $P \subseteq F\left(U \cap M_{i}\right)=$ the Fitting subgroup of $U \cap M_{i}$. If $P \neq F\left(U \cap M_{i}\right)$, then the nontrivial characteristic $p$-complement of $F\left(U \cap M_{i}\right)$ would be normal in $U \cap M_{i}$, hence contained in the $p$-complement $A_{i}$ of $U \cap M_{i}$, which contradicts the fact that $A_{i}$ contains no nontrivial normal subgroup of $U \cap M_{i}$. Thus $P=F\left(U \cap M_{i}\right)$; and since $U \cap M_{i}$ is supersolvable, $\left(U \cap M_{i}\right)^{\prime} \subseteq F\left(U \cap M_{i}\right)=P$, so $\left(U \cap M_{i}\right) / P \simeq A_{i}$ is abelian.

From this it follows that $A=T \cap U \cap M$ is an abelian Hall $\{p, q\}^{\prime}$-subgroup of $M$. Now, $q=\max \left(\pi\left(T \cap M_{i}\right)\right)$. For let $q^{\prime}=\max \left(\pi\left(T \cap M_{i}\right)\right)$; since $T \cap M_{i}$ is supersolvable, it has a normal $q^{\prime}$-Sylow subgroup $Q$. Since the $q$-complement $A_{i}$ of $T \cap M_{i}$ contains no nontrivial normal subgroup of $T \cap M_{i}, Q \nsubseteq A_{i}$, from which it follows that $q^{\prime}=q$. Thus, $q=\max \left(\pi\left(T \cap M_{i}\right)\right)$; in particular, $q \neq \min (\pi(M))$, for otherwise, $T \cap M_{i}$ would be a $q$-group making $M_{i}$ a $\{p, q\}$-group so that each $M_{i}$ and hence $M$ would be solvable.

We thus have that $A$ is an abelian Hall $\{p, q\}^{\prime}$-subgroup of $M$, and $p>q>r$ $=\min (\pi(M))$. Let $R$ be an $r$-Sylow subgroup of $A$, hence of $M ; R$ is abelian. One sees easily that since $R=R^{*} \cap M$ for some $r$-Sylow subgroup $R^{*}$ of $G, N_{G}\left(R^{*}\right)$ $\subseteq N_{G}(R)$ so that $N_{G}(R)$ is abnormal in $G$; and $N_{G}(R) \neq G$ by the minimality of $M$. By hypothesis, therefore, for $N=N_{G}(R), N / \operatorname{scor}_{G} N$ is supersolvable, hence so also is its subgroup $\left(N_{M}\right)\left(\operatorname{scor}_{G} N\right) / \operatorname{scor}_{G} N$, where $N_{M}=N_{M}(R)=M \cap N$. Since $r=$ $\min (\pi(M))$, we see that $N_{M} / N_{M} \cap \operatorname{scor}_{G} N \simeq\left(N_{M}\right)\left(\operatorname{scor}_{G} N\right) / \operatorname{scor}_{G} N$, being supersolvable, has a normal $r$-complement $L / N_{M} \cap \operatorname{scor}_{G} N$.

Since $R \triangleleft N=N_{G}(R)$, we have $R \cap \operatorname{scor}_{G} N \triangleleft \operatorname{scor}_{G} N \triangleleft \triangleleft G$; and because $G$ has no normal solvable subgroups $\neq 1$, and thus no nontrivial subnormal solvable subgroups by Lemma 3.1, $R \cap \operatorname{scor}_{G} N=1$. This means that scor ${ }_{G} N$ is an $r^{\prime}$-group, so $N_{M} \cap \operatorname{scor}_{G} N$ also is, making $L$ a normal $r$-complement of $N_{M}=N_{M}(R)$. It follows that $N_{M}(R)=R \times L$ so that $L$ centralizes $R$; and since $R$ is abelian, we have $R \subseteq Z\left(N_{M}(R)\right)$. However, by Theorem 3 -E, this implies that $M$ is $r$-nilpotent and thus has a proper characteristic subgroup, which contradicts the minimality of $M$.

Consequently, $M$ is solvable; and the result now follows.

An attempt to find more structure in a nonsupersolvable group $G$, structure of the kind given by Doerk in [5], by requiring that the proper normal (or nearly normal, etc.) subgroups also be supersolvable does not seem to be as successful as for nilpotence and $p$-nilpotence in $\$ \S 2$ and 3 . There are some things one can say, among which is the following:

THEOREM 4.7. Let $G$ be a finite nonsupersolvable group having all proper normal subgroups supersolvable. Suppose also that the hypotheses of Corollary 4.5 or of Theorem 4.6 hold. Then, in addition to the conclusions of these results, $G$ has a normal p-Sylow subgroup for $p$ the least or the largest prime factor of $|G|$.

Proof. Let $p=\min (\pi(G))$. Then the proper normal subgroups and subcofactors of the self-normalizing (or abnormal) subgroups of $G$, being supersolvable, are 
$p$-nilpotent. If $G$ is not $p$-nilpotent, then by the results in $\S 3, G$ has a normal $p$ Sylow subgroup. On the other hand, if $G$ is $p$-nilpotent, say with normal $p$-complement $T$, then $T$ is supersolvable by hypothesis. Thus, if $q=\max (\pi(G))=\max (\pi(T))$, then $T$ has a characteristic $q$-Sylow subgroup which is then a normal $q$-Sylow subgroup of $G$.

5. A dual approach. For $H$ a proper subgroup of a finite group $G, \operatorname{cor}_{G} H$ was defined as the maximal $G$-normal subgroup of $H$. We have been considering the effect on $G$ of conditions imposed on $H / \operatorname{cor}_{G} H$ (or $H / \operatorname{scor}_{G} H$ ), where $H$ ranges over a class of proper subgroups of $G$. Now, one might hope to "dualize" this approach by considering for $H<G$, those subgroups which are outside $H$, or at least not contained in $H$, and which are in some sense minimal with respect to the normal structure of $G$. This is the thrust of the remaining three sections.

Following basically the ideas suggested by Deskins in [4], we define for $\mathrm{H}$ a proper subgroup of the finite group $G$, the collection $\mathcal{O}_{G}(H)$ to be the family of subgroups $C$ of $G$ which satisfy the following two conditions: (i) $C \nsubseteq H$; (ii) each proper $G$-normal subgroup of $C$ is contained in $H$. Note that if $C \in \mathcal{O}_{G}(H)$ and $C \triangleleft G$, then $C$ is minimal with respect to being normal in $G$ and not contained in $H$; thus, in a sense, we have dualized the notion of the core of $H$.

In this "outer family" $\mathcal{O}_{G}(H)$, we single out the following subcollections:

(1) $\mathcal{O}_{\triangleleft G}(H)=\left\{C \in \mathcal{O}_{G}(H) \mid C \triangleleft G\right\}$;

(2) $\mathcal{O}_{\phi_{G}}(H)=\left\{C \in \mathcal{O}_{G}(H) \mid C \bowtie G\right\}$;

(3) $\mathcal{O}_{(\text {sn }) G}(H)=\left\{C \in \mathcal{O}_{\phi_{G}}(H) \mid C\right.$ is self-normalizing in $\left.G\right\}$;

(4) $\mathcal{O} \phi_{G}(H)=\left\{C \in \mathcal{O}_{\phi_{G}}(H) \mid C\right.$ is abnormal in $\left.G\right\}$.

Lemma 5.1. Let $H$ be a proper subgroup of the finite group $G, C \in \mathcal{O}_{G}(H)$, and $D$ the maximal G-normal proper subgroup of $C$. Then

(i) $D=\operatorname{cor}_{G}(C \cap H)$.

(ii) If $C \triangleleft G$, then $D=\operatorname{cor}_{G}(C \cap H)=C \cap \operatorname{cor}_{G} H$.

(iii) If $C \Varangle G$, then $D=\operatorname{cor}_{G}(C \cap H)=\operatorname{cor}_{G} C$.

Proof. (i) Since $D$ is normal in $G$ and is properly contained in $C$, we have $D \subseteq H$, so $D \subseteq C \cap H$, and hence $D \subseteq \operatorname{cor}_{G}(C \cap H)$. On the other hand, $\operatorname{cor}_{G}(C \cap H)$ is properly contained in $C$ since $C \cap H \neq C$, so that by the maximality of $D$, $\operatorname{cor}_{G}(C \cap H) \subseteq D$.

(ii) For $C \triangleleft G$, since $C \cap \operatorname{cor}_{G} H$ is a normal subgroup of $G$ contained in $C \cap H$, we have $C \cap \operatorname{cor}_{G} H \subseteq \operatorname{cor}_{G}(C \cap H)$. But $\operatorname{cor}_{G}(C \cap H) \subseteq C \cap H \subseteq C$; and since cor $_{G}(C \cap H)$ is normal in $G$ and is contained in $C \cap H \subseteq H$, we have $\operatorname{cor}_{G}(C \cap H)$ $\subseteq \operatorname{cor}_{G} H$, from which it follows that $\operatorname{cor}_{G}(C \cap H) \subseteq C \cap \operatorname{cor}_{G} H$.

(iii) Since $C \cap H \subseteq C$, the inclusion $\operatorname{cor}_{G}(C \cap H) \subseteq \operatorname{cor}_{G} C$ is immediate. And since $C \bowtie G, \operatorname{cor}_{G} C$ is properly contained in $C$ so that $\operatorname{cor}_{G} C \subseteq H$, hence $\operatorname{cor}_{G} C$ $\subseteq C \cap H$ so that $\operatorname{cor}_{G} C \subseteq \operatorname{cor}_{G}(C \cap H)$.

For $H$ a proper subgroup of the finite group $G$, we define the following outer cofactors of $H$. If $C \in \mathcal{O}_{G}(H)$, we call $C / \operatorname{cor}_{G}(C \cap H)$ an outer cofactor of $H$ in $G$. 
More specifically, for $C \in \mathcal{O}_{\triangleleft}(H)$, we call $C / \operatorname{cor}_{G}(C \cap H)=C / C \cap \operatorname{cor}_{G} H$ a normal outer cofactor of $H$. If $C \in \mathcal{O}_{\triangleleft G}(H)$, we will say that $C / \operatorname{cor}_{G}(C \cap H)=$ $C / \operatorname{cor}_{G} C$ is a nonnormal outer cofactor of $H$; in particular, if $C \in \mathcal{O}_{(s n) G}(H), C / \operatorname{cor}_{G} C$ is a self-normalizing outer cofactor of $H$, and if $C \in \mathcal{O}_{\rtimes_{G}}(H), C / \operatorname{cor}_{G} C$ is an abnormal outer cofactor of $H$.

6. Influence on $G$ of the nonnormal outer cofactors of subgroups. In this section we investigate the properties of a finite group $G$ which arise from conditions imposed on the self-normalizing (or the abnormal) outer cofactors of maximal subgroups of $G$. One such result is given by Deskins in [4]; with the terminology and notation of the preceding section, it can be stated as follows:

THEOREM 6-A. If the finite group $G$ contains a maximal subgroup $S$ which is supersolvable, and if for each $C \in \mathcal{O}_{G}(S)$ with $C \nless G$ or $C \cap S=1, C / \operatorname{cor}_{G}(C \cap S)$ is supersolvable, then $G$ is solvable.

We now establish two general theorems from which results parallel to those in $\S \S 2,3$, and 4 are immediate corollaries. In these two theorems, we assume that the trivial group is always a $\theta$-group, and hence, in particular, that $\theta$-groups do exist.

THEOREM 6.1. Let $\theta$ be a subgroup-inherited homomorphism-invariant property. If the finite group $G$ has a maximal subgroup $S$ such that $S$ and its (1) nonnormal [or (2) self-normalizing, or (3) abnormal] outer cofactors are $\theta$-groups, then $\operatorname{cof}_{G} H=$ $H / \operatorname{cor}_{G} H$ is a $\theta$-group for all (1) nonnormal [or (2) self-normalizing, or (3) abnormal] proper subgroups $H$ of $G$.

Proof. Let $H$ be any proper ( $k$ )-subgroup of $G$, where $k=1,2$, or 3 [that is, $(k)$ denotes any one of the three properties (1) nonnormal, (2) self-normalizing, or (3) abnormal]. If $H \subseteq S$, then trivially $H / \operatorname{cor}_{G} H$ is a $\theta$-group. So suppose $H \nsubseteq S$. If $\operatorname{cor}_{G} H \subseteq S$, then $H$ is an element of $\mathcal{O}_{\phi_{G}}(S), \mathcal{O}_{(s n) G}(S)$, or $\mathcal{O} \triangleleft_{G}(S)$ according as $k=1,2$, or 3 so that, by hypothesis, $H / \operatorname{cor}_{G} H$ is a $\theta$-group. If $\operatorname{cor}_{G} H \nsubseteq S$, then $S\left(\operatorname{cor}_{G} H\right)=G, G / \operatorname{cor}_{G} H=S\left(\operatorname{cor}_{G} H\right) / \operatorname{cor}_{G} H \simeq S / S \cap \operatorname{cor}_{G} H$ is a $\theta$-group, hence so also is its subgroup $H / \operatorname{cor}_{G} H$.

Note. The proof shows that if $S$ and its $(k)$-outer cofactors $C / \operatorname{cor}_{G} C$ with $C$ a maximal subgroup of $G$ are $\theta$-groups, then $H / \operatorname{cor}_{G} H$ is a $\theta$-group for all $(k)$ maximal subgroups of $G$.

The following results are now immediate consequences of this theorem (or the above note), Lemma 5.1, and the corresponding results of $\S \S 2,3$, and 4. Part (b(vi)) strengthens Theorem 6-A by removing the condition "or $C \cap S \neq 1$ " and requiring supersolvability of $C / \operatorname{cor}_{G}(C \cap S)$ for only the self-normalizing $C$ in $\mathcal{O}_{G}(S)$, and by giving information about the Fitting lengths of $G^{\prime}$ and $G$. These results also show that there is nothing especially significant about the condition of supersolvability imposed in Theorem 6-A, but that it can be replaced by a variety of other conditions. 
COROLlary 6.2. Suppose the finite group $G$ has a maximal subgroup $S$ for which one of the following nine conditions holds:

(a) $S$ and all its abnormal outer cofactors $T / \operatorname{cor}_{G} T$ (with $T$ a maximal subgroup of G) are

(i) nilpotent.

(ii) nilpotent of class $\leqq n$.

(iii) solvable of derived length $\leqq n$.

(b) $S$ and all its self-normalizing outer cofactors are

(iv) p-nilpotent.

(v) $\sigma$-Sylow-towered for $\sigma$ some fixed ordering of a set $\Sigma$ of primes containing $\pi(G)$.

(vi) supersolvable.

(c) $S$ and all its abnormal outer cofactors are

(vii) p-nilpotent, and either $p$ is odd or the p-Sylow subgroups of $G$ are abelian.

(viii) $\sigma$-Sylow-towered as in $(\mathrm{b}(\mathrm{v}))$, and the 2-Sylow subgroups of $G$ are abelian.

(ix) supersolvable, and either the 2-Sylow subgroups of $G$ are abelian or the abnormal maximal subgroups of $G$ all have prime-power index.

Then, in the respective cases, the following hold:

(a) (i) $G$ is solvable with $G / F(G)$ nilpotent.

(ii) $G$ is solvable with $G / F(G)$ nilpotent of class $\leqq n$ (and the other results of Theorem 2.3 hold).

(iii) If $G$ is solvable, then $G / F(G)$ has derived length $\leqq n$ (and the other results of Theorem 2.4 hold).

(b) (iv) $G$ has a normal p-subgroup $G / P_{0}$ (which may be trivial) such that $G / P_{0}$ is p-nilpotent; in particular, $G$ is p-solvable of p-length $\leqq 2$.

(v) $G$ is solvable with $G / F(G) \sigma$-Sylow-towered.

(vi) $G$ is solvable with $G / F(G)$ supersolvable and $G / F_{2}(G)$ abelian; thus $f\left(G^{\prime}\right) \leqq 2$ and $f(G) \leqq 3$.

(c) (vii) Same as (b(iv)).

(viii) Same as $(\mathrm{b}(\mathrm{v}))$.

(ix) Same as (b(vi)).

THEOREM 6.3. Let $\theta$ be a subgroup-inherited homomorphism-invariant property. Then, the following are equivalent:

(a) For all abnormal maximal subgroups $S$ of $G$, the (1) self-normalizing [or (2) abnormal] outer cofactors of $S$ are $\theta$-groups.

(b) $H / \operatorname{cor}_{G} H$ is a $\theta$-group for all (1) self-normalizing [or (2) abnormal] proper subgroups $H$ of $G$.

Proof. (b) $\rightarrow$ (a) is immediate. For (a) $\rightarrow$ (b), suppose this to be false, and let $G$ be a minimal counterexample. Then there is some proper (k)-subgroup $H \neq 1$ of $G$ such that $H / \operatorname{cor}_{G} H$ is not a $\theta$-group, where $k=1$ or 2 .

Suppose first that $\operatorname{cor}_{G} H=1$. Then $H \nsubseteq \Gamma(G)=$ the intersection of all abnormal 
maximal subgroups of $G$; for by a result of Gaschütz in [6], $\Gamma(G)$ is a normal nilpotent subgroup of $G$, so if $H \subseteq \Gamma(G)$, then $H \triangleleft \triangleleft \Gamma(G) \triangleleft G$, contradicting the fact that $H$ is a $(k)$-subgroup of $G$. Thus $H \nsubseteq \Gamma(G)$, and there exists an abnormal maximal subgroup $S$ of $G$ not containing $H$. Since $\operatorname{cor}_{G} H=1 \subseteq S, H$ belongs to $\mathcal{O}_{(s n) G}(S)$ or $\mathcal{O}_{\rtimes}(S)$ according as $k=1$ or 2 , so that $H / \operatorname{cor}_{G} H$ is a $\theta$-group by hypothesis. This, however, contradicts the choice of $H$.

Suppose now that $\operatorname{cor}_{G} H \neq 1$, and consider $\bar{G}=G / \operatorname{cor}_{G} H$. We show that (a) holds for $\bar{G}$. For this, let $\bar{S}=S / \operatorname{cor}_{G} H$ be any abnormal maximal subgroup of $\bar{G}$, and let $\bar{C}=C / \operatorname{cor}_{G} H$ be any element of $\mathcal{O}_{(s n) \bar{G}}(\bar{S})$ or $\mathcal{O}_{\triangleleft_{G}}(\bar{S})$, according as $k=1$ or 2 . Then $S$ is an abnormal maximal subgroup of $G$, and $C$ is a $(k)$-subgroup of $G$; also, $C \nsubseteq S$ since $\bar{C} \nsubseteq \bar{S}$. By Lemma $1.3, \operatorname{cor}_{\bar{G}}(\bar{C})=\operatorname{cor}_{G} C / \operatorname{cor}_{G} H$; and $\operatorname{cor}_{\bar{G}}(\bar{C}) \subseteq \bar{S}$ by the definition of an outer cofactor of $\bar{S}$. It follows that $\operatorname{cor}_{G} C \subseteq S$ so that $C$

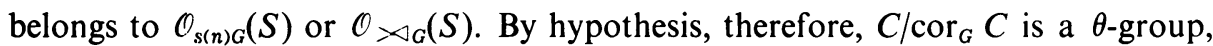
and hence so also is $\bar{C} / \operatorname{cor}_{\bar{G}}(\bar{C}) \simeq C / \operatorname{cor}_{G} G$ (by Lemma 1.3).

Since (a) thus holds for $\bar{G}$ and $|\bar{G}|<|G|$, it follows from the minimality of $G$ that (b) holds for $\bar{G}$. In particular, $\bar{H}=H / \operatorname{cor}_{G} H$ is a proper $(k)$-subgroup of $\bar{G}$ and $\operatorname{cor}_{\bar{G}}(\bar{H})=1$ so that $\bar{H}=\bar{H} / \operatorname{cor}_{\bar{G}}(\bar{H})$ is a $\theta$-group. This, however, again contradicts the choice of $H$.

We conclude, therefore, that no such group $G$ can exist, and the result follows.

Note. An obvious modification of this proof shows that the following also are equivalent: ( $\left.\mathrm{a}^{\prime}\right)$ For all abnormal maximal subgroups $S$ of $G$, the $(k)$-outer cofactors $T / \operatorname{cor}_{G} T$ of $S$ with $T$ a maximal subgroup of $G$ are $\theta$-groups. (b') $H / \operatorname{cor}_{G} H$ is a $\theta$-group for all (k)-maximal subgroups $H$ of $G$.

Corresponding to Corollary 6.2, we have the following results as direct consequences of the preceding theorem (or the above note), Lemma 5.1, and the corresponding results in $\S \S 2,3$, and 4 .

COROLlARY 6.4. Suppose that for each abnormal maximal subgroup $S$ of the finite group $G$, one of the following nine conditions holds:

(a) The abnormal outer cofactors $T / \operatorname{cor}_{G} T$ of $S$ (with $T$ a maximal subgroup of $G$ ) are

(i) nilpotent.

(ii) nilpotent of class $\leqq n$.

(iii) solvable of derived length $\leqq n$.

(b) The self-normalizing outer cofactors of $S$ are

(iv) p-nilpotent.

(v) $\sigma$-Sylow-towered for $\sigma$ some fixed ordering of a set $\Sigma$ of primes containing $\pi(G)$.

(vi) supersolvable.

(c) The abnormal outer cofactors of $S$ are

(vii) p-nilpotent, and either $p$ is odd or the p-Sylow subgroups of $G$ are abelian.

(viii) $\sigma$-Sylow-towered as in $(\mathrm{b}(\mathrm{v}))$, and the 2-Sylow subgroups of $G$ are abelian.

(ix) supersolvable, and either the 2-Sylow subgroups of $G$ are abelian or the abnormal maximal subgroups of $G$ all have prime-power index. 
Then, in the respective cases, the conclusions (a(i)) through (c(ix)) of Corollary 6.2 hold.

7. Influence on $G$ of the normal outer cofactors of subgroups. We consider now the normal outer cofactors of the maximal subgroups of a finite group $G$ and investigate what effect properties imposed on these will have on $G$. Such an approach is suggested by Deskins in [4]. As mentioned there, while every maximal subgroup of a finite solvable group has prime-power index, the converse is not true, as the simple group of order 168 shows. Deskins then defines the normal index of a maximal subgroup; it is precisely this that must be of prime-power for the group to be solvable.

For a maximal subgroup $S$ of $G, \mathcal{O}_{\triangleleft G}(S)$ consists of all those subgroups $H \leqq G$ which satisfy: (1) $H S=G$, (2) $H \triangleleft G$, and (3) $L S=S$ for all proper $G$-normal subgroups $L$ of $H$. The normal outer cofactors of $S$ are the groups $H / \operatorname{cor}_{G}(H \cap S)$ $=H / H \cap \operatorname{cor}_{G} S$ with $H \in \mathcal{O}_{\triangleleft G}(S)$. Using this terminology and notation, we can state the theorem of Deskins, the first part of which makes possible the definition of normal index, as follows:

THEOREM 7-A. Let $S$ be a maximal subgroup of the finite group $G$. Then

(i) All the normal outer cofactors of $S$ have the same order.

(ii) If $[G: S]=$ a power of a prime, then there exists a unique $H$ in $\mathcal{O}_{\triangleleft G}(S)$.

The normal index of a maximal subgroup $S, n(G: S)$, can then be defined as the order of any normal outer cofactor of $S$.

The following theorem extends (i) of Theorem 7-A and also shows that if we impose a condition on one of the normal outer cofactors of a maximal subgroup $S$, then, in fact, we are imposing it on all of them.

THEOREM 7.1. Let $S$ be a maximal subgroup of the finite group $G$. Then all the normal outer cofactors of $S$ are isomorphic.

Proof. Suppose $H$ and $K$ are distinct elements of $\mathcal{O}_{\triangleleft G}(S)$; we are to show that $H / H \cap \operatorname{cor}_{G} S \simeq K / K \cap \operatorname{cor}_{G} S$.

Case 1. $\operatorname{cor}_{G} S=1$.

In this case, $H$ and $K$ are minimal normal in $G$. For if $L \triangleleft G$ with $L \varsubsetneqq H$, then since $H \in \mathcal{O}_{\triangleleft G}(S)$, we have $L \subseteq S$, and hence, $L \subseteq \operatorname{cor}_{G} S=1$. Similarly, $K$ properly contains no nontrivial normal subgroup of $G$. It follows that $H$ and $K$ centralize each other.

Also, $H \cap S=K \cap S=1$. For since $H \triangleleft G, H \cap S \triangleleft S$ so that $S \subseteq N_{G}(H \cap S)$. Now, $K$ centralizes $H \cap S$ since it centralizes $H$, so $K \subseteq N_{G}(H \cap S)$ also. Consesequently, $G=K S \subseteq N_{G}(H \cap S)$, that is, $H \cap S \triangleleft G$. But since $\operatorname{cor}_{G} S=1$, this means that $H \cap S=1$. In the same way, $K \cap S=1$.

Now from Dedekind's law, $H(H K \cap S)=H K \cap H S=H K \cap G=H K$, and $K(H K \cap S)=H K$ also. It follows that $H=H / H \cap K \simeq H K / K=K(K H \cap S) / K$ $\simeq H K \cap S$, and similarly, $K \simeq H K \cap S$. Thus, $H / H \cap \operatorname{cor}_{G} S=H \simeq K=K / K \cap \operatorname{cor}_{G} S$. 
Case 2. $\operatorname{cor}_{G} S \neq 1$.

Consider $\bar{G}=G / \operatorname{cor}_{G} S$, and let $\bar{S}, \bar{H}$, and $\bar{K}$ be the images of $S, H$, and $K$ respectively in $\bar{G}$. Then $\bar{H}$ and $\bar{K}$ belong to $\mathcal{O}_{\triangleleft G}(\bar{S})$. For suppose $\bar{L}=L / \operatorname{cor}_{G} S$ is a normal subgroup of $\bar{G}$ properly contained in $\bar{H}$; then $H \cap L$ is a proper $G$-normal subgroup of $H \in \mathcal{O}_{\triangleleft G}(S)$, so that $H \cap L \subseteq S$, hence $H \cap L \subseteq H \cap \operatorname{cor}_{G} S$. But obviously, the reverse inclusion holds, so $H \cap L=H \cap \operatorname{cor}_{G} S$. It follows that $|H||L| /|H L|$ $=|H \cap L|=\left|H \cap \operatorname{cor}_{G} S\right|=|H|\left|\operatorname{cor}_{G} S\right| /\left|H \operatorname{cor}_{G} S\right|$; and since $H L=H\left(L \operatorname{cor}_{G} S\right)$ $=\left(H \operatorname{cor}_{G} S\right) L=H \operatorname{cor}_{G} S$, we have $|L|=\left|\operatorname{cor}_{G} S\right|$, thus $\bar{L}=1$. This shows that $\bar{H} \in \mathcal{O}_{\triangleleft \bar{G}}(\bar{S})$; similarly, $\bar{K} \in \mathcal{O}_{\triangleleft \bar{G}}(\bar{S})$.

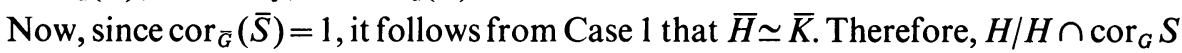
$\simeq \bar{H} \simeq \bar{K} \simeq K / K \cap \operatorname{cor}_{G} S$.

THEOREM 7.2. Let $S$ be a maximal subgroup of the finite group $G$. Then

(i) The normal outer cofactors of $S$ are p-solvable if and only if $n(G: S)$ is a power of $p$ or is prime to $p$.

(ii) The normal outer cofactors of $S$ are solvable if and only if $n(G: S)$ is a power of a prime. In this case, $n(G: S)=[G: S]$.

Proof. (i) Suppose first that the normal outer cofactors of $S$ are $p$-solvable, and let $H \in \mathcal{O}_{\triangleleft G}(S)$. As in the proof of the preceding theorem, $\bar{H}=H\left(\operatorname{cor}_{G} S\right) / \operatorname{cor}_{G} S$ belongs to $\mathcal{O}_{\triangleleft G}(\bar{S})$, where $\bar{G}=G / \operatorname{cor}_{G} S$ and $\bar{S}=S / \operatorname{cor}_{G} S$; and since $\operatorname{cor}_{\bar{G}}(\bar{S})=1$, $\bar{H}$ is minimal normal in $\bar{G}$. By hypothesis, $H / H \cap \operatorname{cor}_{G} S$ is $p$-solvable, hence so also is $\bar{H} \simeq H / H \cap \operatorname{cor}_{G} S$. Thus $n(G: S)=\left|H / H \cap \operatorname{cor}_{G} S\right|=|\bar{H}|$ is either a power of $p$ or is prime to $p$. The converse is an immediate consequence of the definition of $n(G: S)$.

(ii) The equivalence of the two statements follows from (i) and the fact that a finite group is solvable if and only if it is $p$-solvable for all primes $p$ dividing its order. For the second part, let $H \in \mathcal{C}_{\triangleleft G}(S)$ with $H / H \cap \operatorname{cor}_{G} S$ solvable. For $\bar{G}$, $\bar{S}$, and $\bar{H}$ as above, $\bar{H}$ is minimal normal in $G$ and is isomorphic to $H / H \cap \operatorname{cor}_{G} S$ which is solvable, hence $\bar{H}$ is elementary abelian. Now $\bar{H} \cap \bar{S} \triangleleft \bar{S}$ since $\bar{H} \triangleleft \bar{G}$, and $\bar{H} \cap \bar{S} \triangleleft \bar{H}$ since $\bar{H}$ is abelian; thus, $\bar{H} \cap \bar{S} \triangleleft \bar{H} \bar{S}=\bar{G}$. But $\operatorname{cor}_{\bar{G}}(\bar{S})=1$ so that $\bar{H} \cap \bar{S}=1$. Therefore, $[G: S]=[\bar{G}: \bar{S}]=[\bar{H} \bar{S}: \bar{S}]=|\bar{H}|=\left|H / H \cap \operatorname{cor}_{\bar{G}} S\right|=n(G: S)$.

The following lemma will prove useful in induction arguments.

LEMMA 7.3. Let $M$ be a normal subgroup of the finite group $G$, and let $S / M$ be a maximal subgroup of $G / M$. Then each normal outer cofactor of $S / M$ is isomorphic to every normal outer cofactor of $S$.

Proof. Let $\bar{G}=G / M, \bar{S}=S / M$, and let $\bar{K} / \bar{K} \cap \operatorname{cor}_{\bar{G}}(\bar{S})$ be a normal outer cofactor of $\bar{S}$, where $\bar{K}=K / M$. Then $S$ is a maximal subgroup of $G, K \triangleleft G$, and $K \nsubseteq S$. It follows that $L \subseteq K$ for some $L \in \mathcal{O}_{\triangleleft G}(S)$.

Now, $M L=K$. For $M L \subseteq K$ so that $\overline{M L}=M L / M \subseteq K / M=\bar{K}$; also $\overline{M L} \triangleleft \bar{G}$ and $\overline{M L} \nsubseteq \bar{S}$ since $L \nsubseteq S$. Thus, since $\bar{K} \in \mathcal{O}_{\triangleleft \bar{G}}(\bar{S})$, we have $\overline{M L}=\bar{K}$, hence, $M L=K$. 
Since $M \subseteq \operatorname{cor}_{G} S$, it follows that

$$
\begin{aligned}
L / L \cap \operatorname{cor}_{G} S & \simeq L\left(\operatorname{cor}_{G} S\right) / \operatorname{cor}_{G} S=L M\left(\operatorname{cor}_{G} S\right) / \operatorname{cor}_{G} S=K\left(\operatorname{cor}_{G} S\right) / \operatorname{cor}_{G} S \\
& \simeq K / K \cap \operatorname{cor}_{G} S ;
\end{aligned}
$$

and from Lemmas 5.1 and 1.3, we have

$$
\begin{aligned}
K / K \cap \operatorname{cor}_{G} S & =K / \operatorname{cor}_{G}(K \cap S) \\
& \simeq(K / M) /\left(\operatorname{cor}_{G}(K \cap S) / M\right)=\bar{K} / \operatorname{cor}_{G}(\bar{K} \cap \bar{S})=\bar{K} / \bar{K} \cap \operatorname{cor}_{G}(\bar{S}) .
\end{aligned}
$$

Therefore, the normal outer cofactor $\bar{K} / \bar{K} \cap \operatorname{cor}_{\bar{G}}(\bar{S})$ of $\bar{S}=S / M$ is isomorphic to the normal outer cofactor $L / L \cap \operatorname{cor}_{G} S$ of $S$, and hence, by Theorem 7.1, to every normal outer cofactor of $S$.

In [4], Deskins states the following equivalences:

THEOREM 7-B. For $G$ a finite group, the following are equivalent:

(i) $G$ is solvable.

(ii) Each maximal subgroup of $G$ has prime-power normal index.

(iii) The index and normal index are equal for each maximal subgroup of $G$.

Beidleman and Spencer in [2] extend the equivalence of (i) and (iii), and also establish some additional results as given in the following theorem:

THEOREM 7-C. (a) The finite group $G$ is p-solvable if and only if $(n(G: S))_{p}=$ $[G: S]_{p}$ for each maximal subgroup $S$ of $G$, where $k_{p}$ denotes the p-part of the integer $k$.

(b) If $G$ has a solvable maximal subgroup with prime-power normal index, then $G$ is solvable.

(c) If $G$ has a solvable maximal subgroup $S$ with $n(G: S)=[G: S]$, then $G$ is solvable.

The following theorem gives some additional equivalences to the $p$-solvability of $G$ and provides extensions of (i) $\leftrightarrow$ (ii) in Theorem 7-B and of (b) of Theorem 7-C, plus a slight extension of (a) of Theorem 7-C.

THEOREM 7.4. For $G$ a finite group, the following are equivalent:

(i) $G$ is p-solvable.

(ii) $G$ has a maximal subgroup $S$ such that $S$ and its normal outer cofactors are p-solvable.

(iii) $G$ has a p-solvable maximal subgroup $S$ with $n(G: S)$ a power of $p$ or prime to $p$.

(iv) For each abnormal maximal subgroup $S$ of $G$, the normal outer cofactors of $S$ are p-solvable.

(v) For each abnormal maximal subgroup $S$ of $G, n(G: S)$ is a power of $p$ or is prime to $p$.

(vi) For each abnormal maximal subgroup $S$ of $G,(n(G: S))_{p}=[G: S]_{p}$.

[Note. As the proof will show, the word "abnormal" can be omitted in any of (iv) through (vi).] 
Proof. The equivalence of (ii) and (iii) as well as that of (iv) and (v) is immediate from Theorem 7.2.

(i) $\leftrightarrow$ (ii). Since (i) $\rightarrow$ (ii) is obvious, only the converse requires proof. For this, let $S$ be a $p$-solvable maximal subgroup of $G$ with $p$-solvable normal outer cofactor $K / K \cap \operatorname{cor}_{G} S$. We use induction on $|G|$, and consider two cases.

Case 1. $K \cap \operatorname{cor}_{G} S=1$.

In this case, $K$ is minimal normal in $G$; for if the normal subgroup $L$ of $G$ is properly contained in $K$, then since $K \in \mathcal{O}_{\triangleleft G}(S)$, it follows that $L \subseteq K \cap S$, and thus $L \subseteq \operatorname{cor}_{G}(K \cap S)=K \cap \operatorname{cor}_{G} S=1$. Now, $K=K / K \cap \operatorname{cor}_{G} S$ is $p$-solvable; and since $K S=G$, we have $G / K=K S / K \simeq S / S \cap K$ is $p$-solvable by the $p$-solvability of $S$, so the $p$-solvability of $G$ follows.

Case 2. $K \cap \operatorname{cor}_{G} S \neq 1$.

Let $M$ be a minimal normal subgroup of $G$ contained in $K \cap \operatorname{cor}_{G} S$. From the solvability of $S, M$ is $p$-solvable. Now, $S / M$ is a $p$-solvable maximal subgroup of $G / M$, and by Lemma 7.3 , the normal outer cofactors of $S / M$ are $p$-solvable. By induction, therefore, $G / M$ is $p$-solvable, and the $p$-solvability of $G$ follows.

(i) $\leftrightarrow$ (iv). Only (iv) $\rightarrow$ (i) requires proof, since the converse is obvious. For this, if $G$ is simple, then every maximal subgroup of $G$ is abnormal in $G$. Let $S$ be any such, and let $H \in \mathcal{O}_{\triangleleft G}(S)$. Then $H \nsubseteq S, H \triangleleft G$, and $G$ simple imply that $H=G$, so $\mathcal{O}_{\triangleleft G}(S)=\{G\}$. Therefore, since $\operatorname{cor}_{G} S=1, G=G / G \cap \operatorname{cor}_{G} S$ is $p$-solvable.

So suppose that $G$ is not simple, and let $M$ be a minimal normal subgroup. Then $M$ is $p$-solvable. For if $M \subseteq \Gamma(G)=$ the intersection of all abnormal maximal subgroups of $G$, then since $\Gamma(G)$ is nilpotent (Gaschütz [6]), $M$ is nilpotent, hence $p$-solvable. If $M \nsubseteq \Gamma(G)$, then there is some abnormal maximal subgroup $S$ not containing $M$; then $M \in \mathcal{O}_{\triangleleft G}(S)$ so that $M=M / M \cap \operatorname{cor}_{G} S$ is $p$-solvable by hypothesis.

Now consider $G / M$. If $S / M$ is any abnormal maximal subgroup of $G / M$, then $S$ is an abnormal maximal subgroup of $G$, hence has $p$-solvable normal outer cofactors. By Lemma 7.3, the normal outer cofactors of $S / M$ are also $p$-solvable. Thus, the hypotheses hold for $G / M$ so that, by induction, $G / M$ is $p$-solvable. The $p$-solvability of $G$ now follows.

(i) $\leftrightarrow$ (vi). The implication (i) $\rightarrow$ (vi) is a consequence of the Beidleman-Spencer theorem (Theorem 7-C). For the converse, if $G$ is simple, then each maximal subgroup of $G$ is abnormal, so the $p$-solvability of $G$ follows from this same result. So suppose $G$ is not simple, and let $M$ be a minimal normal subgroup. Using Lemma 7.3, we have by induction that $G / M$ is $p$-solvable. If $G$ has a minimal normal subgroup $M^{*} \neq M$, then $G / M^{*}$ also is $p$-solvable, and the $p$-solvability of $G=G / M \cap M^{*}$ follows.

So assume that $M$ is the unique minimal normal subgroup of $G$; we need only show that $M$ is $p$-solvable. If $M \subseteq \Phi(G)$, this is immediate; so suppose there exist maximal subgroups not containing $M$. Let $S$ be any such; by the uniqueness of $M$, $S$ is abnormal in $G$. We may assume that $p|| M \mid$. Now, $n(G: S)=|M|$, so, by 
hypothesis, $[G: S]_{p}=(n(G: S))_{p}=|M|_{p}$ so that $p \mid[G: S]$. Thus $M \subseteq \phi_{p}(G)=$ the intersection of all maximal subgroups of $G$ with $[G: S]$ prime to $p$. By a result of Deskins in [4], $\phi_{p}(G)$ is solvable, so $M$ is solvable.

In the following theorem, most of the equivalences are immediate corollaries of Theorem 7.4; the equivalence of (i) and (iv) is part (c) of Theorem 7-C, and is included here for completeness.

THEOREM 7.5. For $G$ a finite group, the following are equivalent:

(i) $G$ is solvable.

(ii) $G$ has a maximal subgroup $S$ such that $S$ and its normal outer cofactors are solvable.

(iii) $G$ has a solvable maximal subgroup $S$ with $n(G: S)$ a power of a prime.

(iv) $G$ has a solvable maximal subgroup $S$ with $n(G: S)=[G: S]$.

(v) For each abnormal maximal subgroup $S$ of $G$, the normal outer cofactors of $S$ are solvable.

(vi) For each abnormal maximal subgroup $S$ of $G, n(G: S)$ is a power of a prime.

(vii) For each abnormal maximal subgroup $S$ of $G, n(G: S)=[G: S]$.

(viii) For each abnormal maximal subgroup $S$ of $G, K \cap S$ is normal in $G$ for all $K \in \mathcal{O}_{\triangleleft G}(S)$.

(ix) For each abnormal maximal subgroup $S$ of $G, K \cap S$ is subnormal in $G$ for all $K \in \mathcal{O}_{\triangleleft G}(S)$.

[Note. As in Theorem 7.4, the word "abnormal" can be omitted in any of (v)-(ix).]

Proof. The equivalence of (i), (ii), (iii), (v), (vi), and (vii) is immediate from Theorem 7.4 and the fact that a finite group is solvable if and only if it is $p$-solvable for all primes $p$ dividing its order. The equivalence of (i) and (iv) is the result of Beidleman and Spencer (part (c) of Theorem 7-C).

(vii) $\leftrightarrow$ (viii). Let $S$ be a maximal subgroup of $G$ and $K \in \mathcal{O}_{\triangleleft G}(S)$. If $K \cap S \triangleleft G$, then $K \cap \operatorname{cor}_{G} S=\operatorname{cor}_{G}(K \cap S)=K \cap S$, thus

$$
n(G: S)=\left|K / K \cap \operatorname{cor}_{G} S\right|=[K: K \cap S]=[K S: S]=[G: S] .
$$

For the converse, this argument is reversible. For if $n(G: S)=[G: S]$, then

$$
|K| K \cap \operatorname{cor}_{G} S \mid=[G: S]=[K S: S]=[K: K \cap S]
$$

thus since $K \cap \operatorname{cor}_{G} S \subseteq K \cap S$, we have, using Lemma 5.1, that

$$
K \cap S=K \cap \operatorname{cor}_{G} S=\operatorname{cor}_{G}(K \cap S),
$$

so $K \cap S \triangleleft G$.

(viii) $\leftrightarrow$ (ix). We show first that if $T$ is any subgroup of $G$ and $H \in \mathcal{O}_{\triangleleft G}(T)$, then $\operatorname{scor}_{G}(H \cap T)=H \cap \operatorname{scor}_{G} T$. For this, since $H \triangleleft G$ and $\operatorname{scor}_{G} T \triangleleft \triangleleft G$, we have $H \cap \operatorname{scor}_{G} T \triangleleft \triangleleft G$, hence $H \cap \operatorname{scor}_{G} T \subseteq \operatorname{scor}_{G}(H \cap T)$. On the other hand, since $\operatorname{scor}_{G}(H \cap T)$ is contained in $T$ and is subnormal in $G$, it must be contained in $\operatorname{scor}_{G} T$; consequently, $\operatorname{scor}_{G}(H \cap T) \subseteq H \cap \operatorname{scor}_{G} T$. 
Now let $S$ be a maximal subgroup of $G$ and let $K \in \mathcal{O}_{\triangleleft G}(S)$. From the above and Lemma 1.2, $\operatorname{scor}_{G}(K \cap S)=K \cap \operatorname{scor}_{G} S=K \cap \operatorname{cor}_{G} S=\operatorname{cor}_{G}(K \cap S)$. Thus, since $K \cap S \triangleleft \triangleleft G$ if and only if $\operatorname{scor}_{G}(K \cap S)=K \cap S$, it follows that $K \cap S \triangleleft \triangleleft G$ if and only if $K \cap S=\operatorname{cor}_{G}(K \cap S)$ which is equivalent to $K \cap S$ being normal in $G$.

Our last two results extend the following theorem of Deskins in [4]:

THEOREM 7-D. The intersection of those maximal subgroups $S$ of the finite group $G$ with $n(G: S)$ divisible by two distinct primes is the largest normal solvable subgroup of $G$ (the intersection of an empty collection of subgroups being understood, as usual, to be all of $G$ ).

THEOREM 7.6. Let $p$ be a prime dividing $|G|, R_{p}(G)=$ the largest normal p-solvable subgroup of $G$, and let $\mathscr{M}_{p}(G)=$ the collection of maximal subgroups $S$ of $G$ with $n(G: S)$ divisible by both $p$ and some prime distinct from $p$, or equivalently, those maximal subgroups $S$ with non-p-solvable normal outer cofactors. Then

$$
R_{p}(G)=\bigcap\left\{S \mid S \in \mathscr{M}_{p}(G)\right\}=\bigcap\left\{S \in \mathscr{M}_{p}(G) \mid S \triangleleft \triangleleft\right\} .
$$

Proof. Letting $T_{p}(G)=\bigcap\left\{S \mid S \in \mathscr{M}_{p}(G)\right\}$, and $T_{p}^{*}(G)=\bigcap\left\{S \in \mathscr{M}_{p}(G) \mid S \rtimes \triangleleft\right\}$, we show (i) $R_{p}(G)=T_{p}(G)$, and then (ii) $R_{p}(G)=T_{p}^{*}(G)$.

(i) We note first that every $p$-solvable minimal normal subgroup $M$ of $G$ (which is thus either a $p$-group or a $p^{\prime}$-group) is contained in $T_{p}(G)$. For suppose $M \nsubseteq T_{p}(G)$ for such an $M$; then there exists $S \in \mathscr{M}_{p}(G)$ with $M \nsubseteq S$, so $M \nsubseteq \operatorname{cor}_{G} S$. Now, $M \in \mathcal{O}_{\triangleleft G}(S)$, so $n(G: S)=|M| M \cap \operatorname{cor}_{G} S|=| M \mid$; hence, $|M|$ is divisible by both $p$ and some prime $\neq p$, which contradicts $M$ being $p$-solvable and minimal normal in $G$.

It follows then that if $T_{p}(G)=1$, then $R_{p}(G)=1$ also. We may assume, therefore, that $T_{p}(G) \neq 1$. Now, $T_{p}(G) \triangleleft G$. For from the definition $\mathcal{O}_{\triangleleft G}(S)$ for $S$ a maximal subgroup of $G$, if $x \in G$ then $H \in \mathcal{O}_{\triangleleft G}(S)$ if and only if $H \in \mathcal{O}_{\triangleleft G}\left(S^{x}\right)$; thus, $n(G: S)$ $=n\left(G: S^{x}\right)$ so that $S \in \mathscr{M}_{p}(G)$ if and only if $S^{x} \in \mathscr{M}_{p}(G)$.

Let $M$ be a minimal normal subgroup of $G$ contained in $T_{p}(G)$. We show first that $M$ is $p$-solvable, hence is either a $p$-group or a $p^{\prime}$-group and is contained in $R_{p}(G)$. For suppose $M$ is not $p$-solvable. Then $M \nsubseteq \Gamma(G)$, so there exists an abnormal maximal subgroup $S$ of $G$ not containing $M$. Then $M \in \mathcal{O}_{\triangleleft G}(S)$ and $n(G: S)=|M|$. Since $M$ is not $p$-solvable, there exists a prime $q \neq p$ such that both $p$ and $q$ divide $|M|$. Then both $p$ and $q$ divide $n(G: S)=|M|$, so $S \in \mathscr{M}_{p}(G)$; and since $M \nsubseteq S$, this means that $M \nsubseteq T_{p}(G)$, a contradiction.

Now, $R_{p}(G / M)=R_{p}(G) / M$. For clearly, $R_{p}(G) / M \subseteq R_{p}(G / M)$; and if $R_{p}(G) / M$ $=K / M$, then $K \triangleleft G$ and $K$ is $p$-solvable from the $p$-solvability of $K / M$ and $M$, so $K \subseteq R_{p}(G)$, hence, $R_{p}(G / M)=K / M \subseteq R_{p}(G) / M$.

Also, we have $T_{p}(G / M)=T_{p}(G) / M$. For by Lemma 7.3 , if $S / M$ is a maximal subgroup of $G / M$, then $n(G / M: S / M)=n(G: S)$; and since $M \subseteq T_{p}(G), M \subseteq S$ for all $S \in \mathscr{M}_{p}(G)$. It follows that $S \in \mathscr{M}_{p}(G)$ if and only if $S / M \in \mathscr{M}_{p}(G / M)$. Thus $\mathscr{M}_{p}(G / M)$ $=\left\{S / M \mid S \in \mathscr{M}_{p}(G)\right\}$, so $T_{p}(G / M)=\bigcap\left\{S / M \mid S \in \mathscr{M}_{p}(G)\right\}=\left[\bigcap\left\{S \mid S \in \mathscr{M}_{p}(G)\right\}\right] / M$ $=T_{p}(G) / M$. 
Now, by induction, $R_{p}(G / M)=T_{p}(G / M)$; therefore, by what we have just shown, $R_{p}(G)=T_{p}(G)$.

(ii) Obviously, $T_{p}(G) \subseteq T_{p}^{*}(G)$; thus, if $T_{p}^{*}(G)=1$, then $T_{p}(G)=1$, so from (i), we have $T_{p}^{*}(G)=R_{p}(G)=1$. So suppose $T_{p}^{*}(G) \neq 1 . T_{p}^{*}(G)$ is normal in $G$; for from the proof of (i), if $x \in G$, then $S \in \mathscr{M}_{p}(G)$ if and only if $S^{x} \in \mathscr{M}_{p}(G)$, and clearly, $S \triangleleft G$ if and only if $S^{x}>\triangleleft G$.

Let $M$ be a minimal normal subgroup of $G$ contained in $T_{p}^{*}(G)$. From (i), $R_{p}(G / M)=R_{p}(G) / M$. Also, we have $T_{p}^{*}(G / M)=T_{p}^{*}(G) / M$. For letting $\mathscr{M}_{p}^{*}(G)$ be the collection $\left\{S \in \mathscr{M}_{p}(G) \mid S \triangleleft G\right\}$, we have from the proof of (i) that $\mathscr{M}_{p}^{*}(G / M)$ is the collection $\left\{S / M \mid S / M-\triangleleft G / M\right.$ and $\left.S \in \mathscr{M}_{p}(G)\right\}$; and since $S / M$ is an abnormal maximal subgroup of $G / M$ if and only if $S$ is an abnormal maximal subgroup of $G$ containing $M$, we have $\mathscr{M}_{p}^{*}(G / M)=\left\{S / M \mid S \in \mathscr{M}_{p}^{*}(G)\right.$ and $\left.M \subseteq S\right\}$. But $M \subseteq T_{p}^{*}(G)$, hence $M \subseteq S$ for all $S \in \mathscr{M}_{p}^{*}(G)$, so $\mathscr{M}_{p}^{*}(G / M)=\left\{S / M \mid S \in \mathscr{M}_{p}^{*}(G)\right\}$. It now follows that $T_{p}^{*}(G / M)=T_{p}^{*}(G) / M$.

By induction, $R_{p}(G / M)=T_{p}^{*}(G / M)$; thus, by what we have just shown, $R_{p}(G)$ $=T_{p}^{*}(G)$.

Corollary 7.7. Let $R(G)$ be the largest normal solvable subgroup of $G$, and $\mathscr{M}(G)=$ the collection of all maximal subgroups $S$ of $G$ with $n(G: S)$ divisible by two distinct primes, or equivalently, those maximal subgroups with nonsolvable normal outer cofactors. Then $R(G)=\bigcap\{S \mid S \in \mathscr{M}(G)\}=\bigcap\{S \in \mathscr{M}(G) \mid S \rtimes G\}$.

Proof. Let $\pi(G)=\left\{p_{1}, \ldots, p_{m}\right\}$. From the fact that a finite group is solvable if and only if it is $p$-solvable for all primes $p$ dividing its order, it follows that $R(G)$ $=\bigcap_{i=1}^{m} R_{p_{i}}(G)$, where $R_{p_{i}}(G)$ is the largest normal $p_{i}$-solvable subgroup of $G$. Now, for each $i=1, \ldots, m$, if $\mathscr{M}_{p_{i}}(G)=\left\{S_{i j} \mid j=1, \ldots, n_{i}\right\}$ is the collection of maximal subgroups with normal index divisible by poth $p_{i}$ and some prime $\neq p_{i}$, then from Theorem 7.6 we obtain $R(G)=\bigcap_{i=1}^{m} R_{p_{i}}(G)=\bigcap_{i=1}^{m} \bigcap_{j=1}^{n_{1}} S_{i j}=\bigcap\{S \mid S \in \mathscr{M}(G)\}$. In a similar manner, $R(G)=\bigcap\{S \in \mathscr{M}(G) \mid S \ngtr G\}$.

\section{REFERENCES}

1. R. Baer, Classes of finite groups and their properties, Illinois J. Math. 1 (1957), 115-187. MR 19, 386.

2. J. Beidleman and A. Spencer, The normal index of maximal subgroups in finite groups, Illinois J. Math. (submitted).

3. W. E. Deskins, A condition for the solvability of a finite group, Illinois J. Math. 5 (1961), 306-313. MR 23 \#A1708.

4. —_, On maximal subgroups, Proc. Sympos. Pure Math., vol. 1, Amer. Math. Soc., Providence, R. I., 1959, pp. 100-104. MR 23 \#A2462.

5. K. Doerk, Minimal nicht überauflösbare, endliche Gruppen, Math. Z. 91 (1966), 198-205. MR 33 \#189.

6. W. Gaschütz, Uber die Ф-Untergruppe endlicher Gruppen, Math. Z. 58 (1953), 160-170. MR 15, 285.

7. D. Gorenstein, Finite groups, Harper \& Row, New York, 1968. MR 38 \#229.

8. P. Hall, On the system normalizers of a soluble group, Proc. London Math. Soc. 43 (1937), $507-528$. 
9. B. Huppert, Endliche Gruppen. I, Die Grundlehren der math. Wissenschaften, Band 134, Springer-Verlag, Berlin, 1967. MR 37 \#302.

10. —_ Normalteiler und maximale Untergruppen endlicher Gruppen, Math. Z. 60 (1954), 409-434. MR 16, 332.

11. N. Itô, Note on (LM)-groups of finite orders, Kōdai Math. Sem. Rep. 1951, 1-6. MR 13, 317.

12. K. Iwasawa, Uber die Struktur der endlichen Gruppen, deren echte Untergruppen sämtlich nilpotent sind, Proc. Phys.-Math. Soc. Japan (3) 23 (1941), 1-4. MR 2, 211.

13. J. S. Rose, Finite groups with prescribed Sylow tower subgroups, Proc. London Math. Soc. (3) 16 (1966), 577-589. MR 33 \#5734.

14. - The influence on a finite group of its proper abnormal structure, J. London Math. Soc. 40 (1965), 348-361. MR 30 \#4838.

15. O. Schmidt, Über Gruppen, deren sämtliche Teiler spezielle Gruppen sind, Rec. Math. Moscow 31 (1924), 366-372.

16. W. R. Scott, Group theory, Prentice-Hall, Englewood Cliffs, N. J., 1964. MR 29 \#4785.

\section{Calvin College,}

Grand Rapids, Michigan 49506 